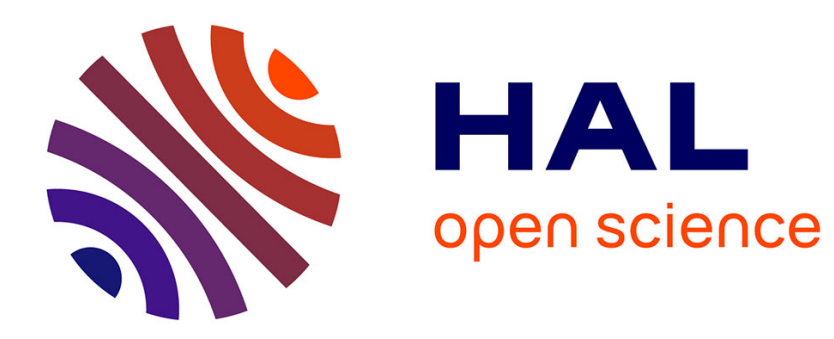

\title{
A classification of Tsirelson type spaces
}

Jordi Lopez Abad, Antonis Manoussakis

\section{To cite this version:}

Jordi Lopez Abad, Antonis Manoussakis. A classification of Tsirelson type spaces. 2005. hal00012301

\section{HAL Id: hal-00012301 \\ https://hal.science/hal-00012301}

Preprint submitted on 19 Oct 2005

HAL is a multi-disciplinary open access archive for the deposit and dissemination of scientific research documents, whether they are published or not. The documents may come from teaching and research institutions in France or abroad, or from public or private research centers.
L'archive ouverte pluridisciplinaire HAL, est destinée au dépôt et à la diffusion de documents scientifiques de niveau recherche, publiés ou non, émanant des établissements d'enseignement et de recherche français ou étrangers, des laboratoires publics ou privés. 


\title{
A CLASSIFICATION OF TSIRELSON TYPE SPACES
}

\author{
J. LOPEZ-ABAD AND A. MANOUSSAKIS
}

\begin{abstract}
We give a complete classification of mixed Tsirelson spaces $T\left[\left(\mathcal{F}_{i}, \theta_{i}\right)_{i=1}^{r}\right]$ for finitely many pairs of given compact and hereditary families $\mathcal{F}_{i}$ of finite sets of integers and $0<\theta_{i}<1$ in terms of the Cantor-Bendixson indexes of the families $\mathcal{F}_{i}$, and $\theta_{i}(1 \leq i \leq r)$. We prove that there are unique countable ordinal $\alpha$ and $0<\theta<1$ such that every block sequence of $T\left[\left(\mathcal{F}_{i}, \theta_{i}\right)_{i=1}^{r}\right]$ has a subsequence equivalent to a subsequence of the natural basis of the $T\left(\mathcal{S}_{\omega^{\alpha}}, \theta\right)$. Finally, we give a complete criterion of comparison in between two of these mixed Tsirelson spaces.
\end{abstract}

\section{INTRODUCTION}

The line of research we continue in this paper has been initiated by an old problem of S. Banach asking if every Banach space contains a subspace isomorphic to $c_{0}$ or some $\ell_{p}$. This problem was solved negatively by B. S. Tsirelson [20] who provided the first example of a Banach space that does not contain any of the spaces $c_{0}, \ell_{p}, 1 \leq p<\infty$. The idea of Tsirelson's construction became particularly apparent after T. Figiel and W. B. Johnson 112 have shown that the norm of the dual of Tsirelson space satisfy the following implicit equation

$$
\left\|\sum_{n} a_{n} e_{n}\right\|=\max \left\{\sup _{n}\left|a_{n}\right|, \frac{1}{2} \sup \sum_{i=1}^{d}\left\|E_{i}\left(\sum_{n} a_{n} e_{n}\right)\right\|\right\}
$$

where the sequences $\left(E_{i}\right)_{i=1}^{d}$ considered above consists on successive subsets of integers with the property that $d \leq \min E_{1}, d \in \mathbb{N}$, and $E_{i}\left(\sum_{n} a_{n} e_{n}\right)=\sum_{n \in E_{i}} a_{n} e_{n}$ is the restriction of $\sum_{n} a_{n} e_{n}$ on the set $E_{i}$. We refer to [11] for an extended study of Tsirelson space $T$. A first systematic abstract study on Tsirelson construction was given by S. Bellenot [8] and S. A. Argyros and I. Deliyanni [4]. Given a real number $0<\theta<1$ and an arbitrary compact and hereditary family $\mathcal{F}$ of finite sets of integers one defines the Tsirelson type Banach space $T(\mathcal{F}, \theta)$ as the completion of $c_{00}$ with the implicitly given norm (1) replacing $1 / 2$ by $\theta$ and using sequences $\left(E_{i}\right)_{i}$ of finite sets of integers which are $\mathcal{F}$-admissible, i.e. there is some $\left\{m_{i}\right\}_{i=1}^{d} \in \mathcal{F}$ such that $m_{1} \leq \min E_{1} \leq \max E_{1}<m_{2} \leq \min E_{2} \leq \max E_{2}<\cdots<m_{d} \leq \min E_{d} \leq \max E_{d}$. In this notation, Tsirelson example is the space $T(\mathcal{S}, 1 / 2)$, where $\mathcal{S}=\{s \subseteq \mathbb{N}: \# s \leq \min s\}$ is the

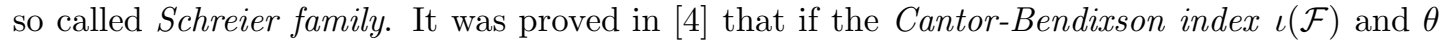
satisfy the inequality $\theta \cdot \iota(\mathcal{F})>1$, then the space $T(\mathcal{F}, \theta)$ is reflexive. Moreover, in the case of $\iota(\mathcal{F}) \geq \omega$, they proved that the space $T(\mathcal{F}, \theta)$ does not contain any of the classical spaces $c_{0}$ or $\ell_{p}, 1 \leq p<\infty$. In the case that $\mathcal{F}$ is chosen to be the family of the finite subsets of $\mathbb{N}$ with cardinality at most $n \geq 2$, denoted by $[\mathbb{N}]^{\leq n}$, it was shown in $[8]$, [4 that the corresponding space $T([\mathbb{N}] \leq n, \theta)$ is isomorphic to $c_{0}$ if $n \theta \leq 1$ and is isomorphic to $\ell_{p}(1<p<\infty)$ if $\theta=n^{-1 / q}$, where $q$ is the conjugate of $p$ (i.e. $1 / p+1 / q=1$ ).

Further examples of Tsirelson type spaces with interesting properties are the spaces $T\left(\mathcal{S}_{\alpha}, \theta\right)$ considered in [1], , [3], where the compact and hereditary families $\mathcal{S}_{\alpha}$ are the $\alpha$-Schreier families, the natural generalizations of the Schreier family to index $\omega^{\alpha}\left(\mathcal{S}_{1}=\mathcal{S}\right)$. These spaces share many properties with the original Tsirelson space, and their natural Schauder bases are examples of $w$-null sequences with large oscillation indexes. A basic property of any $\mathcal{S}_{\alpha}$ is that it is spreading (see definition below). This is used to show that every normalized block sequence with respect to their natural bases $\left(e_{n}\right)$ is equivalent to a subsequence of $\left(e_{n}\right)$, a property that

Research partially supported by EПEAK program Pythagoras. 
$c_{0}$ and $\ell_{p}$ also have. From this, and the fact that the Cantor-Bendixson indexes of the families $\mathcal{S}_{\alpha}$ and $[\mathbb{N}] \leq n$ are very much different, it can be explained why $T\left(\mathcal{S}_{\alpha}, 1 / 2\right)$ does not contain isomorphic copies of $\ell_{p} \cong T\left([\mathbb{N}]^{\leq n}, n^{-1 / q}\right)$ or $c_{0} \cong T([\mathbb{N}] \leq n, 1 / n)$.

The aim of this paper is to understand in these terms the so called mixed Tsirelson spaces $T\left[\left(\mathcal{F}_{i}, \theta_{i}\right)_{i=1}^{r}\right]$, whose norms are defined implicitly by

$$
\|x\|_{\left(\mathcal{F}_{i}, \theta_{i}\right)_{i=1}^{r}}=\max \left\{\|x\|_{\infty}, \sup \left\{\theta_{i} \sum_{j=1}^{n}\left\|E_{j} x\right\|_{\left(\mathcal{F}_{i}, \theta_{i}\right)_{i=1}^{r}}:\left(E_{j}\right)_{j=1}^{n} \text { is } \mathcal{F}_{i} \text {-admissible, } 1 \leq i \leq r\right\}\right\},
$$

for arbitrary compact and hereditary families $\mathcal{F}_{i}$ and establish a criterion of comparability in between them. The first step in this direction was done by J. Bernues and I. Deliyanni [9] and J. Bernues and J. Pascual [10] who proved the following two results:

a) If the Cantor-Bendixson indexes of the families are finite then $T\left[\left(\mathcal{F}_{i}, \theta_{i}\right)_{i=1}^{r}\right]$ is saturated by either to $c_{0}$ or to some $\ell_{p}, 1<p<\infty$.

b) If the Cantor Bendixson index of $\mathcal{F}$ is equal to $\omega+1$ then $T(\mathcal{F}, \theta)$ contains a subspace isomorphic to a subspace of $T(\mathcal{S}, \theta)$.

The only case left is when one of the families has infinite index. Recall that every ordinal $\alpha>0$ has a unique decomposition as $\alpha=\omega^{\beta} k+\delta$, where $\delta<\omega^{\beta}$ and $k \in \mathbb{N}$. Using it twice it follows that every infinite ordinal $\alpha$ has the unique decomposition $\alpha=\omega^{\omega^{\gamma} n+\xi} m+\eta$ (see 14). Now given a compact family $\mathcal{F}$, let $\gamma(\mathcal{F})$ and $n(\mathcal{F})$ be $\omega^{\omega^{\gamma}}$ and $n$ in the previous decomposition for $\alpha$ equal to the Cantor-Bendixson index of $\mathcal{F}$. Following this notation, our main result is the following

Theorem. Fix $\left(\mathcal{F}_{i}, \theta_{i}\right)_{i=1}^{r}$ such that at least one of the families has infinite index. Then there is some $1 \leq i_{0} \leq r$ such that for every compact and hereditary family $\mathcal{G}$ the following are equivalent.

(1) $\gamma(\mathcal{G})=\gamma\left(\mathcal{F}_{i_{0}}\right)$.

(2) Every infinite dimensional closed subspace of $T\left[\left(\mathcal{F}_{i}, \theta_{i}\right)_{i=1}^{r}\right]$ contains a subspace isomorphic to a subspace of $T\left(\mathcal{G}, \theta_{i_{0}}^{n(\mathcal{G}) / n\left(\mathcal{F}_{i_{0}}\right)}\right)$.

(3) Every normalized block sequence $\left(x_{n}\right)$ of $T\left[\left(\mathcal{F}_{i}, \theta_{i}\right)_{i=1}^{r}\right]$ has a subsequence $\left(x_{n}\right)_{n \in M}$ equivalent to the subsequence $\left(e_{\min \operatorname{supp}} x_{n}\right)_{n \in M}$ of the basis of $T\left(\mathcal{G}, \theta_{i_{0}}^{n(\mathcal{G}) / n\left(\mathcal{F}_{i_{0}}\right)}\right)$.

It readily follows that

(c) every normalized block sequence $\left(x_{n}\right)$ of $T\left[\left(\mathcal{F}_{i}, \theta_{i}\right)_{i=1}^{r}\right]$ has a subsequence $\left(x_{n}\right)_{n \in M}$ equivalent to the subsequence $\left(e_{\min \operatorname{supp} x_{n}}\right)_{n \in M}$ of the basis of $T\left[\left(\mathcal{F}_{i}, \theta_{i}\right)_{i=1}^{r}\right]$.

(d) There are unique countable ordinal $\alpha$ and $0<\theta<1$ such that every normalized block sequence with respect to the basis $\left(e_{n}\right)$ of $T\left[\left(\mathcal{F}_{i}, \theta_{i}\right)_{i=1}^{r}\right]$ has a subsequence equivalent to a subsequence of the basis $\left(e_{n}\right)$ of $T\left(\mathcal{S}_{\omega^{\alpha}}, \theta\right)$.

So, for example $T\left(\mathcal{S}_{\omega^{3} 4+\omega 5}, 1 / 2^{4}\right)$ and $T\left(\mathcal{S}_{\omega^{3}}, 1 / 2\right)$ are mutually saturated, while $T\left(\mathcal{S}_{\omega^{3}}, 1 / 2\right)$ and $T\left(\mathcal{S}_{\omega^{4}}, 1 / 2\right)$ are totally incomparable.

Another consequence is that every subspace of $T\left[\left(\mathcal{F}_{i}, \theta_{i}\right)_{i=1}^{r}\right]$ contains a $\mathcal{S}_{\omega^{\alpha}}-\ell_{1}$ spreading model, that is, there exits a constant $K>1$ such that for every sequence of coefficients $\left(a_{n}\right)_{n}$

$$
\left\|\sum_{n \in s} a_{n} x_{n}\right\| \geq \frac{1}{K} \sum_{n \in s}\left|a_{n}\right| \quad\left(s \in \mathcal{S}_{\omega^{\alpha}}\right)
$$

In particular, every subspace of $T\left[\left(\mathcal{F}_{i}, \theta_{i}\right)_{i=1}^{r}\right]$ contains an asymptotic $\ell_{1}$-subspace. Asymptotic $\ell_{1}$-spaces, the structure of these spaces as well as the structure of the spreading models of a Banach space is a current research topic, which provides interesting examples and structural results in Banach space theory (see [2], [18]).

The proofs given in this paper use four main ingredients: We work with the equivalent reformulation of the implicit norm of $T\left[\left(\mathcal{F}_{i}, \theta_{i}\right)_{i=1}^{r}\right]$ given by the norming set $K\left(\left(\mathcal{F}_{i}, \theta_{i}\right)_{i=1}^{r}\right)$, and the so-called tree analysis of a functional of $K(\mathcal{F}, \theta)$ (see section 1 ). In particular, given a normalized block sequence $\left(x_{n}\right)$ of the basis $\left(e_{n}\right)$ we provide an algorithm to estimate the norm of a linear combination $\sum_{n} a_{n} x_{n}$ in terms of a corresponding linear combination of a 
subsequence of the basis $\left(e_{n}\right)$ of an auxiliary space $T\left[\left(\mathcal{G}_{i}, \theta_{i}\right)_{i=1}^{r}\right]$, much in the spirit of wellknown works in this field. Secondly, we use the well know fact (see [13]), [7]) that given two compact and hereditary families $\mathcal{F}$ and $\mathcal{G}$ there is an infinite set $M$ such that either $\mathcal{F} \mid N=$ $\{s \in \mathcal{F}: s \subseteq N\} \subseteq \mathcal{G} \mid N=\{s \in \mathcal{G}: s \subseteq N\}$ or viceversa. This is indeed a consequence of the fact that for every compact and hereditary family $\mathcal{F}$ there is an infinite set $M$ such that $\mathcal{F} \mid M$ is, what we call here, homogeneous on $M$. It turns out that the $\subseteq$-maximal elements of such families have the Ramsey property, which we will use here to avoid some combinatorial computations.

Finally, we reduce the study of $T\left[\left(\mathcal{F}_{i}, \theta_{i}\right)_{i=1}^{r}\right]$ for compact and hereditary families $\mathcal{F}_{i}$ 's to the case of $T(\mathcal{G}, \theta)$ for some regular family $\mathcal{G}$, i.e. a compact hereditary family $\mathcal{G}$ that is in addition spreading (see below). This additional regularity property of families $\mathcal{G}$ have two main advantages; the first is that the associated norming set $K(\mathcal{G}, \theta)$ has a simpler form; the second one is that their Cantor-Bendixson index is preserved if we restrict them to an infinite set.

The paper is organized as follows: In the first section we introduce notation, basic combinatorial definitions, and mixed Tsirelson spaces. In the second section we study the behavior of subsequences of the natural basis of $T\left[\left(\mathcal{F}_{i}, \theta_{i}\right)_{i=1}^{r}\right]$ in the case of regular families. An important outcome of this section is the reduction we make from finitely many families to one.

The third section is devoted to an abstract study of compact and hereditary families of finite sets of integers. In particular, we introduce homogeneous and uniform families and we prove two combinatorial results, basic tools for this work. This section provide us the link between mixed Tsirelson spaces built by compact and hereditary families with Tsirelson type spaces constructed using a regular family.

In the last section we show that every block sequence of a mixed Tsirelson space $T\left[\left(\mathcal{F}_{i}, \theta_{i}\right)_{i=1}^{r}\right]$ has a further subsequence equivalent to a subsequence of its basis. As a consequence of this and of the results of the previous sections we provide several saturation results. We give also, using special convex combinations, two criteria to obtain incomparability for Tsirelson type spaces. Finally, we expose the classification of mixed Tsirelson spaces $T\left[\left(\mathcal{F}_{i}, \theta_{i}\right)_{i=1}^{r}\right]$.

Acknowledgments. We thank to S. A. Argyros and S. Todorcevic for their useful remarks.

\section{BASIC FACTS}

Thorough all this paper we are going to deal with families of finite sets of integers. The family of all finite sets of integers is denoted here by FIN. Given $s, t \in$ FIN we write $s<t$ (resp. $s \leq t$ ) to denote that $\max s<\min t($ resp. $\max s \leq \min t$ ), and for an integer $n$ we write $n<s(n \leq s)$ whenever $\{n\}<s($ resp. $\{n\} \leq s)$. These orders can be easily extended to vectors $x, y \in c_{00}(\mathbb{N}): x<y(x \leq y)$ iff $\operatorname{supp} x<\operatorname{supp} y($ resp. $\operatorname{supp} x \leq \operatorname{supp} y)$, where for $x \in c_{00}, \operatorname{supp} x=\{n \in \mathbb{N}: x(n) \neq 0\}$. We say that a sequence $\left(s_{n}\right)$ of finite sets of integers is a block sequence if $s_{n}<s_{n+1}$ for every $n$. In a similar manner one defines the corresponding notion of block sequence of vectors of $c_{00}$.

Given an infinite set $M$ and a finite set $s$ we denote $M / s=\{n \in M: n>s\}$, and for a given integer $n$, let $I / n=I /\{n\}$. The shift of a non-empty set $A$ of integers is ${ }_{*} A=A \backslash \min A$. Given two sets $A$ and $B$ we set $A \backslash B=\{n \in A: n \notin B\}$, and $M \backslash m=\{n \in M: n \geq m\}$. For a given family $\mathcal{F} \subseteq$ FIN, an infinite set $M \subseteq \mathbb{N}$ and a finite set $s$, let $\mathcal{F} \mid M=\{s \in \mathcal{F}: s \subseteq M\}$ be the restriction of $\mathcal{F}$ in $M$, and let $\mathcal{F}_{s}=\{t \in \mathrm{FIN}: s<t, s \cup t \in \mathcal{F}\}$. Given a finite set $s$ we use \#s to denote its cardinality. Finally, every time we write an enumeration $A=\left\{m_{i}\right\}$ of a set $A$ we mean an strictly increasing enumeration.

Concerning now in topological aspects, observe that the family of all finite sets of integers has the natural topology induced by the product topology on the Cantor space $\{0,1\}^{\mathbb{N}}$, simply by identifying every finite set $s$ with its characteristic function $\xi_{s}: \mathbb{N} \rightarrow\{0,1\}$. We say then that a family $\mathcal{F} \subseteq$ FIN is compact if $\mathcal{F}$ is closed with respect to the previous topology. This means that there is no infinite sequence $\left(s_{n}\right) \subseteq \mathcal{F}$ such that $s_{n} \varsubsetneqq s_{n+1}$. Given a compact family $\mathcal{F}$, recall that $\partial \mathcal{F}$ is the set of all proper accumulation points of $\mathcal{F}$ and that $\partial^{(\alpha)}(\mathcal{F})=\bigcap_{\beta<\alpha} \partial\left(\partial^{(\beta)}(\mathcal{F})\right)$. The rank is well defined since $\mathcal{F}$ is countable and therefore a scattered compactum, so the 
sequence $\left(\partial^{(\alpha)}(\mathcal{F})\right)_{\alpha}$ of iterated derivatives must vanish. We define, as in [9], the CantorBendixson index $\iota(\mathcal{F})$ of a compact family $\mathcal{F}$ as the minimal ordinal $\alpha$ such that $\partial^{(\alpha)} \mathcal{F} \subseteq\{\emptyset\}$. Observe that this definition is a slight variation of the standard one, where one considers the first ordinal $\alpha$ such that $\partial^{(\alpha)} \mathcal{F}$ vanishes. Let us point out the reason to take this definition of the index of a family $\mathcal{F}$ : while for families with infinite index the results we present in this paper have exactly the same form using the standard notion of Cantor-Bendixson index, for families with finite index the standard Cantor-Bendixon index cannot be used to characterize the corresponding mixed Tsirelson spaces (see [9]).

A family $\mathcal{F}$ is called hereditary iff $s \subseteq t \in \mathcal{F}$ implies that $s \in \mathcal{F}$. Another relevant order of FIN is $\preceq$ : Given two finite sets $s$ and $t$ we write $s \preceq t$ iff $|t|=|s|$ and the only strictly increasing map $\sigma: t \rightarrow s$ satisfies that $n \geq \sigma(n)$ for all $n \in t$, or equivalently if $s=\left\{s_{1}, \ldots, s_{d}\right\}$ then $t=\left\{t_{1}, \ldots, t_{d}\right\}$ and $s_{i} \leq t_{i}$ for every $i \leq d$. We say that a family $\mathcal{F}$ of finite subsets of an infinite set $M$ is spreading on $M$ if $s \preceq t \subseteq M$ and $s \in \mathcal{F}$ implies $t \in \mathcal{F}$. We say that $\mathcal{F}$ is spreading if it is spreading on $\mathbb{N}$. We say that $\mathcal{F}$ is regular on $M$ iff it is compact hereditary and spreading on $M$, and that $\mathcal{F}$ is regular if it is regular on $\mathbb{N}$.

Examples of regular families are the families of subsets of $M$ with cardinality $\leq n$, denoted by $[M]^{\leq n}$, and with index $n$. Indeed, we will see that every regular family with finite index is, when restricted to some tail $\mathbb{N} / n$, of this form (see Proposition 3.4). A regular family of index $\omega$ is the well-known Schreier family

$$
\mathcal{S}=\{s \in \mathrm{FIN}: \# s \leq \min s\} .
$$

In general, for a countable ordinal $\alpha$ we can define inductively on $\alpha$ an $\alpha$-Schreier family by $\mathcal{S}_{1}=\mathcal{S}, \mathcal{S}_{\alpha+1}=\left\{s_{1} \cup \cdots \cup s_{n}:\left(s_{i}\right) \subseteq \mathcal{S}_{\alpha}\right.$ is $\mathcal{S}$-admissible $\}$, and if $\alpha$ is a limit ordinal, $\mathcal{S}_{\alpha}=\bigcup_{n} \mathcal{S}_{\alpha_{n}} \uparrow(\mathbb{N} \backslash n)$, where $\left(\alpha_{n}\right)$ is a fixed increasing sequence of ordinals with limit $\alpha$. It can be shown that $\mathcal{S}_{\alpha}$ is a regular family with index $\omega^{\alpha}$ 迎. We introduce now two well known operations between families of finite sets.

Definition 1.1. Fix two families $\mathcal{F}$ and $\mathcal{G}$ of finite sets. Recall the following from [0]

$$
\begin{aligned}
& \mathcal{F} \oplus \mathcal{G}=\{s \cup t: s<t, s \in \mathcal{G}, t \in \mathcal{F}\}, \\
& \mathcal{F} \otimes \mathcal{G}=\left\{s_{1} \cup \cdots \cup s_{n}:\left(s_{i}\right) \text { is block, } s_{i} \in \mathcal{F} \text { and }\left\{\min s_{i}\right\} \in \mathcal{G}\right\} .
\end{aligned}
$$

The operation $\mathcal{F} \oplus \mathcal{G}$ is called block sum while the operation $\mathcal{F} \otimes \mathcal{G}$ is called convolution. Observe that $\alpha+1$-Schreier families are defined inductively by the formula $\mathcal{S}_{\alpha+1}=\mathcal{S}_{\alpha} \otimes \mathcal{S}$. Also, it is well known that the index of the families $\mathcal{F} \oplus \mathcal{G}$ and $\mathcal{F} \otimes \mathcal{G}$ are equal $\iota(\mathcal{F})+\iota(\mathcal{G})$ and $\iota(\mathcal{F}) \iota(\mathcal{G})$ respectively, assuming that $\mathcal{F}, \mathcal{G}$ are regular (see Proposition 3.4). So, if $\alpha$ has Cantor normal form $\alpha=\omega^{\alpha_{0}} n_{0}+\cdots+\omega^{\alpha_{k}} n_{k}$ (see 14 for standard properties of ordinal arithmetic), the regular family $\left(\mathcal{S}_{\alpha_{0}} \otimes[\mathbb{N}] \leq n_{0}\right) \oplus \cdots \oplus\left(\mathcal{S}_{\alpha_{k}} \otimes[\mathbb{N}] \leq n_{k}\right)$ is of index $\alpha$.

It is not difficult to prove that $\oplus$ and $\otimes$ share many properties with the addition and multiplication of ordinals. For example, $\oplus$ and $\otimes$ are associative, and they have the distributive law $\mathcal{F} \otimes(\mathcal{G} \oplus \mathcal{H})=(\mathcal{F} \otimes \mathcal{G}) \oplus(\mathcal{F} \otimes \mathcal{H})$, while in general the two operations are not commutative or $(\mathcal{F} \oplus \mathcal{G}) \otimes \mathcal{H} \neq(\mathcal{F} \otimes \mathcal{H}) \oplus(\mathcal{G} \otimes \mathcal{H})$ (as for the addition and multiplication of ordinals).

In order to keep the notation easier we introduce the following notation

Notation. By $\left(\mathcal{F}_{i}, \theta_{i}\right)_{i \in I}$ we shall mean a sequence of pairs of compact and hereditary families $\mathcal{F}_{i}$ and real numbers $0<\theta_{i}<1(i \in I)$. We call a sequence $\left(\mathcal{F}_{i}, \theta_{i}\right)_{i \in I}$ regular if in addition every $\mathcal{F}_{i}$ is regular. Given two sequences $\left(\mathcal{F}_{i}, \theta_{i}\right)_{i \in I}$ and $\left(\mathcal{F}_{i}, \theta_{i}\right)_{i \in J}$ we use $\left(\mathcal{F}_{i}, \theta_{i}\right)_{i \in I} \frown\left(\mathcal{F}_{i}, \theta_{i}\right)_{i \in J}$ to denote the concatenation of the two sequences $\left(\mathcal{F}_{i}, \theta_{i}\right)_{i \in I \cup J}$. Given $\mathcal{F} \subseteq$ FIN and $m \in \mathbb{N}$ let

$$
\mathcal{F}^{\otimes(m)}=\mathcal{F} \otimes \stackrel{(m)}{\cdots} \otimes \mathcal{F}
$$

We are now ready to give the definition of mixed Tsirelson spaces. 
Definition 1.2. Given a sequence $\left(\mathcal{F}_{i}, \theta_{i}\right)_{i \in I}$ the norm $\|\cdot\|_{\left(\mathcal{F}_{i}, \theta_{i}\right)_{i \in I}}$ on $c_{00}$ is defined as follows. For $x \in c_{00}$ let

$$
\|x\|_{\left(\mathcal{F}_{i}, \theta_{i}\right)_{i \in I}}=\max \left\{\|x\|_{\infty}, \sup \left\{\theta_{i} \sum_{j=1}^{n}\left\|E_{j} x\right\|_{\left(\mathcal{F}_{i}, \theta_{i}\right)_{i \in I}}:\left(E_{j}\right)_{j=1}^{n} \text { is } \mathcal{F}_{i^{-}} \text {-admissible, } i \in I\right\}\right\} .
$$

Next, $T\left[\left(\mathcal{F}_{i}, \theta_{i}\right)_{i \in I}\right]$ denotes the completion of $\left(c_{00},\|\cdot\|_{\left(\mathcal{F}_{i}, \theta_{i}\right)_{i=1}^{r}}\right)$. Observe that a Tsirelson type space $T(\mathcal{F}, \theta)$ is nothing else but the mixed Tsirelson space $T[(\mathcal{F}, \theta)]$.

REMARK 1.3. (a) From the hereditariness of the families $\mathcal{F}_{i}(i \in I)$ it follows easily that the Hammel standard basis $\left(e_{n}\right)$ of $c_{00}$ is an 1-unconditional normalized Schauder basis of $T\left[\left(\mathcal{F}_{i}, \theta_{i}\right)_{i \in I}\right]$. In the sequel whenever we consider block sequences will be with respect the basis $\left(e_{n}\right)_{n}$.

(b) The basis $\left(e_{n}\right)$ is also boundedly complete, and if there exists $i \in I$ with $\theta_{i}>1 / \iota\left(\mathcal{F}_{i}\right)$ (with the convention $1 / \iota\left(\mathcal{F}_{i}\right)=0$ for $\iota\left(\mathcal{F}_{i}\right)$ is infinite) then $\left(e_{n}\right)$ is also shrinking. Therefore in this case $T\left[\left(\mathcal{F}_{i}, \theta_{i}\right)_{i \in I}\right]$ is reflexive (see [] for more details).

(c) Observe that if in the previous definition of the norm $\|\cdot\|_{\left(\mathcal{F}_{i}, \theta_{i}\right)_{i \in I}}$ we do not impose that $\mathcal{F}_{i}$ are necessarily hereditary but only $\sqsubseteq$-hereditary $(s \sqsubseteq t$ if $s \subseteq t$ and $s<t \backslash s)$ then in the corresponding completion $T\left[\left(\mathcal{F}_{i}, \theta_{i}\right)_{i \in I}\right]$ the sequence $\left(e_{n}\right)$ is still a bimonotone Schauder basis, not necessarily unconditional.

(d) It can be shown that the implicit formula (2) remains true for every $x \in T\left[\left(\mathcal{F}_{i}, \theta_{i}\right)_{i \in I}\right]$ (see [16] or Remark 1.4 below).

(e) If we allow to some of the families $\mathcal{F}_{i}$ to be non-compact, i.e. some of their closures contain an infinite set, then it follows easily that $T\left[\left(\mathcal{F}_{i}, \theta_{i}\right)_{i=1}^{r}\right]$ is $\ell_{1}$-saturated. Indeed, every seminormalized block sequence contains a further subsequence, for which every finite initial subsequence is $\mathcal{F}_{i}$-admissible for a non-compact family $\mathcal{F}_{i}$, and hence equivalent to the natural basis of $\ell_{1}$.

We present now an standard alternative description of the norm of the space $T\left[\left(\mathcal{F}_{i}, \theta_{i}\right)_{i \in I}\right]$, closer to the spirit of Tsirelson's original definition. Let us denote by $K\left(\left(\mathcal{F}_{i}, \theta_{i}\right)_{i \in I}\right)$ the minimal subset of $c_{00}$

(a) containing $\pm e_{n}^{*}(n \in \mathbb{N})$

(b) it is closed under the $\left(\mathcal{F}_{i}, \theta_{i}\right)$-operation $(i \in I): \theta\left(f_{1}+\cdots+f_{n}\right) \in K\left(\left(\mathcal{F}_{i}, \theta_{i}\right)_{i \in I}\right)$ for every $\mathcal{F}_{i}$-admissible sequence $\left(f_{i}\right)_{i=1}^{n} \subseteq K\left(\left(\mathcal{F}_{i}, \theta_{i}\right)_{i \in I}\right)$.

The norm induced by $K\left(\left(\mathcal{F}_{i}, \theta_{i}\right)_{i \in I}\right)$, i.e.

$$
\|x\|_{K\left(\left(\mathcal{F}_{i}, \theta_{i}\right)_{i \in I}\right)}=\sup \left\{f(x): f \in K\left(\left(\mathcal{F}_{i}, \theta_{i}\right)_{i \in I}\right)\right\} \text {, for } x \in c_{00},
$$

is exactly the norm $\|x\|_{\left(\mathcal{F}_{i}, \theta_{i}\right)_{i \in I}}$ defined above. Given an infinite set $M$ of integers we set

$$
\left.K^{M}\left(\left(\mathcal{F}_{i}, \theta_{i}\right)_{i \in I}\right)=\left\{\phi \in K\left(\left(\mathcal{F}_{i}, \theta_{i}\right)_{i \in I}\right): \operatorname{supp} \phi \subseteq M\right\}\right\} .
$$

REMARK 1.4. (a) It is easy to see that the closure under the pointwise convergence topology of conv $K\left(\left(\mathcal{F}_{i}, \theta_{i}\right)_{i \in I}\right)$ is the unit dual ball $B_{T\left[\left(\mathcal{F}_{i}, \theta_{i}\right)_{i \in I}\right]^{*}}$. It follows that $B_{T\left[\left(\mathcal{F}_{i}, \theta_{i}\right)_{i \in I}\right]^{*}}$ is closed under the $\left(\mathcal{F}_{i}, \theta_{i}\right)$-operation $(i \in I)$.

(b) For every infinite set $M$ of integers and every sequence $\left(a_{n}\right)_{n \in M}$ of scalars we have

$$
\left\|\sum_{n \in M} a_{n} e_{n}\right\|_{\left(\mathcal{F}_{i}, \theta_{i}\right)_{i \in I}}=\left\|\sum_{n \in M} a_{n} e_{n}\right\|_{K^{M}\left(\left(\mathcal{F}_{i}, \theta_{i}\right)_{i \in I}\right)} .
$$

Observe that $K^{M}\left(\left(\mathcal{F}_{i}, \theta_{i}\right)_{i \in I}\right)=K\left(\left(\mathcal{F}_{i} \mid M, \theta_{i}\right)_{i \in I}\right)$ if $\mathcal{F}_{i}$ is regular for every $i \in I$, but that in general the previous inequality is not true.

Notice that, by minimality of $K\left(\left(\mathcal{F}_{i}, \theta_{i}\right)_{i \in I}\right)$, every functional from $K\left(\left(\mathcal{F}_{i}, \theta_{i}\right)_{i \in I}\right)$ either has the form $\pm e_{n}^{*}(n \in \mathbb{N})$, or it is the result of a $\left(\mathcal{F}_{i}, \theta_{i}\right)$-operation to some sequence in $K\left(\left(\mathcal{F}_{i}, \theta_{i}\right)_{i \in I}\right)$ and $i \in I$. This suggests that somehow every element of $K\left(\left(\mathcal{F}_{i}, \theta_{i}\right)_{i \in I}\right)$ has a complexity, that increases in every use of the $\left(\mathcal{F}_{i}, \theta_{i}\right)$-operations. This is captured by the following definition. 
Definition 1.5. [5] A family $\left(f_{t}\right)_{t \in \mathcal{T}} \subseteq K\left(\left(\mathcal{F}_{i}, \theta_{i}\right)_{i \in I}\right)$ is called a tree analysis of a functional $f \in K\left(\left(\mathcal{F}_{i}, \theta_{i}\right)_{i \in I}\right)$ if the following are satisfied:

(i) $\mathcal{T}=\left(\mathcal{T}, \preceq_{\mathcal{T}}\right)$ is a finite tree with a unique root denoted by $\emptyset$, and $f_{\emptyset}=f$.

(ii) For every $t \in \mathcal{T}$ maximal node, $f_{t}=\varepsilon_{t} e_{k_{t}}^{*}$ where $\varepsilon_{t}= \pm 1$.

(iii) For every $t \in \mathcal{T}$ which is not maximal, there exists $i \in I$ such that $\left(f_{s}\right)_{s \in S_{t}}$ is $\mathcal{F}_{i}$-admissible and $f_{t}=\theta_{i} \sum_{s \in S_{t}} f_{s}$, where $S_{t}$ denotes the set of immediate successor nodes of $t$.

Note that $S_{t}$ is well ordered by $s_{0}<s_{1}$ iff $\operatorname{supp} f_{s_{0}}<\operatorname{supp} f_{s_{1}}$. Whenever there is no possible confusion we will write $\preceq$ in order to denote $\preceq_{\mathcal{T}}$.

It is not difficult to see, by the minimality of the set $K\left(\left(\mathcal{F}_{i}, \theta_{i}\right)_{i \in I}\right)$, that every functional of $K\left(\left(\mathcal{F}_{i}, \theta_{i}\right)_{i \in I}\right)$ admits a tree analysis.

As we mentioned before in Remark 1.4, in general it is not true that $K^{M}\left(\left(\mathcal{F}_{i}, \theta_{i}\right)_{i \in I}\right)=$ $K\left(\left(\mathcal{F}_{i} \backslash M, \theta_{i}\right)_{i \in I}\right)$ for a given infinite set $M$ of integers, so, a priori, it does not suffice to control the restrictions $\mathcal{F}_{i} \backslash M(i \in I)$ for the understanding of norms $\left\|\sum_{n \in M} a_{n} e_{n}\right\|_{\left(\mathcal{F}_{i}, \theta_{i}\right)_{i \in I}}$. We will see soon that the following is a key definition for this purpose.

Definition 1.6. Given a family $\mathcal{F}$ we define the family of all $\mathcal{F}$-admissible sets as follows: We say that a finite set $t=\left\{m_{i}\right\}_{i=0}^{k-1}$ interpolates the block sequence $\left(s_{i}\right)_{i=0}^{k-1}$ of finite sets iff

$$
m_{0} \leq s_{0}<m_{1} \leq s_{1}<\cdots<m_{n-1} \leq s_{n-1} .
$$

We say that $t=\left\{n_{i}\right\}$ interpolates $s=\left\{m_{i}\right\}$ iff $t$ interpolates the block sequence $\left(\left\{m_{i}\right\}\right)$.

Given a family $\mathcal{F}$ of finite sets, a block sequence $\left(s_{i}\right)_{i=0}^{n-1}$ of finite sets is $\mathcal{F}$-admissible if there is some $t \in \mathcal{F}$ which interpolates $\left(s_{i}\right)_{i=0}^{n-1}$. We define

$$
\operatorname{Ad}(\mathcal{F})=\left\{\left\{m_{i}\right\}_{i=0}^{n} \in \mathrm{FIN}:\left(\left\{m_{i}\right\}\right)_{i=0}^{n} \text { is } \mathcal{F} \text {-admissible }\right\},
$$

the family of all $\mathcal{F}$-admissible finite sets.

Notice that if $M \subseteq \mathbb{N}$ and $\left(s_{i}\right)$ is an $\mathcal{F}$-admissible sequence of subsets of $M$, then $\left\{\min s_{i}\right\} \in$ $\operatorname{Ad}(\mathcal{F})\lceil M$. The converse is not true in general.

We list some properties of the $\mathcal{F}$-admissible. Particularly interesting is the characterization of spreadness of a family in terms of its $\mathcal{F}$-admissible sets.

Proposition 1.7. (a) $\mathcal{F} \subseteq \operatorname{Ad}(\mathcal{F})$.

(b) If $\mathcal{F}$ is compact or hereditary, then so is $\operatorname{Ad}(\mathcal{F})$.

(c) $\mathcal{F}$ is spreading on $M$ iff $\operatorname{Ad}(\mathcal{F}) \mid M=\mathcal{F}$.

(d) Set $\operatorname{Ad}^{(n+1)}(\mathcal{F})=\operatorname{Ad}\left(\operatorname{Ad}^{(n)} \mathcal{F}\right), \operatorname{Ad}^{(0)}(\mathcal{F})=\mathcal{F}$. Then $\operatorname{spread}(\mathcal{F})=\{s: \exists t \in \mathcal{F}(t \preceq s)\}=$ $\bigcup_{n} \operatorname{Ad}^{(n)}(\mathcal{F})$ is the minimal spreading family on $\mathbb{N}$ containing $\mathcal{F}$. In case that $\mathcal{F}$ is compact or hereditary then so is $\operatorname{spread}(\mathcal{F})$, and if $\mathcal{F}$ is regular on some set $M, \operatorname{spread}(\mathcal{F})\lceil M=\mathcal{F}$.

Proof. (a), (b) are easily proved.

(c): If $\mathcal{F}$ is spreading on $M$, and $t \in \mathcal{F}$ interpolates some $s \subseteq M$, then, in particular, $t \preceq s$ and hence $s \in \mathcal{F}$. Suppose that $\operatorname{Ad}(\mathcal{F})=\mathcal{F}$, and suppose that $s \preceq t$, with $s \in \mathcal{F}$ and $t \subseteq M$. Set $s=\left\{m_{i}\right\}_{i=1}^{k}$ and $t=\left\{n_{i}\right\}_{i=1}^{k}$. For each $0 \leq j \leq k$ let $t_{j}=\left\{m_{i}: 1 \leq i \leq k-j\right\} \cup\left\{n_{i}\right.$ : $k-j+1 \leq i \leq k\}$. Observe that $t_{0}=s \in \mathcal{F}, t_{j}$ interpolates $t_{j+1}$ and that $t_{k}=t$, so an easy inductive argument finishes the proof of (c). (d) follows by similar arguments than $(c)$.

Finally, let us recall the following from 13

Theorem 1.8. Suppose that $\mathcal{F}$ and $\mathcal{G}$ are two compact and hereditary families. Then there is some infinite set $M$ such that either $\mathcal{F}|M \subseteq \mathcal{G}| M$ or $\mathcal{G}|M \subseteq \mathcal{F}| M$.

As for regular families $\mathcal{F}$ we have that $\iota(\mathcal{F} \mid M)=\iota(\mathcal{F})$ for every $M$ (see Proposition 3.4), it follows that if $\mathcal{F}$ and $\mathcal{G}$ are two regular families with $\iota(\mathcal{F})<\iota(\mathcal{G})$ then for every $M$ there is some $N \subseteq M$ such that $\mathcal{F}|N \subseteq \mathcal{G}| N$. In other words, strict inequalities between indexes of regular families imply, modulo restrictions, strict inclusion between those families. 


\section{Subsequences of THE BASIS FOR REGUlar FAMILIES.}

The purpose of this section is to understand, for regular families, the relationship between the operations $\oplus$ and $\otimes$ on regular families and corresponding norming sets. For example, what is the relation between $K(\mathcal{F} \oplus \mathcal{F}, \theta)$ and $K(\mathcal{F}, \theta)$ ? It is well known that if the family $\mathcal{F}$ has finite index, then these two norming sets are, in general, different, as the corresponding Tsirelson type spaces are isomorphic to different $\ell_{p}$ 's. However if $\mathcal{F}$ is, for example, the Schreier family $\mathcal{S}$ then it can be easily shown that $[\mathbb{N}] \leq 3 \otimes \mathcal{S} \subseteq \mathcal{S} \otimes[\mathbb{N}]^{\leq 2}$, and hence

$$
[\mathbb{N}]^{\leq 8} \otimes\left(\mathcal{S} \otimes[\mathbb{N}]^{\leq 2}\right) \subseteq\left([\mathbb{N}]^{\leq 3} \otimes\left([\mathbb{N}]^{\leq 3} \otimes \mathcal{S}\right)\right) \otimes[\mathbb{N}]^{\leq 2} \subseteq\left(\mathcal{S} \otimes[\mathbb{N}]^{\leq 4}\right) \otimes[\mathbb{N}]^{\leq 2}=\mathcal{S} \otimes[\mathbb{N}]^{\leq 8}
$$

It follows, by induction on the complexity of $\phi \in K\left(\mathcal{S} \otimes[\mathbb{N}]^{\leq 2}, \theta\right)$ that $\phi=\phi_{1}+\cdots+\phi_{8}$ for some block sequence $\left(\phi_{i}\right)_{i=1}^{8} \subseteq K(\mathcal{S}, \theta)$. This clearly implies that

$$
\left\|\sum_{n} a_{n} e_{n}\right\|_{(\mathcal{S}, \theta)} \leq\left\|\sum_{n} a_{n} e_{n}\right\|_{(\mathcal{S} \otimes[\mathbb{N}] \leq 2, \theta)} \leq 8\left\|\sum_{n} a_{n} e_{n}\right\|_{(\mathcal{S}, \theta)}
$$

for every $0<\theta<1$ and every sequence $\left(a_{n}\right)$ of scalars. As one can guess this reasoning cannot be applied to an arbitrary regular family $\mathcal{F}$ with infinite index since we do not have an explicit presentation of $\mathcal{F}$ as for the Schreier family. However, we do have the index of the family, and by the properties of the ordinals we have that

$$
\iota\left([\mathbb{N}]^{\leq 3} \otimes(\mathcal{F} \otimes[\mathbb{N}] \leq 2)\right)=3(\iota(\mathcal{F}) 2)<\iota(\mathcal{F}) 2+\omega \leq \iota(\mathcal{F}) 3
$$

and, since $\mathcal{F}$ is regular, there is some infinite set $M$ of integers such that $[M] \leq 3 \otimes(\mathcal{F} \mid M \otimes$ $[M] \leq 2) \subseteq \mathcal{F} \otimes[\mathbb{N}] \leq 3$, hence

$$
\left\|\sum_{n \in M} a_{n} e_{n}\right\|_{(\mathcal{F}, \theta)} \leq\left\|\sum_{n \in M} a_{n} e_{n}\right\|_{(\mathcal{F} \otimes[\mathbb{N}] \leq 2, \theta)} \leq 3\left\|\sum_{n \in M} a_{n} e_{n}\right\|_{(\mathcal{F}, \theta)},
$$

so the two subsequences $\left(e_{n}\right)_{n \in M} \subseteq T(\mathcal{F}, \theta)$ and $\left(e_{n}\right)_{n \in M} \subseteq T\left(\mathcal{F} \otimes[\mathbb{N}]^{\leq 2}, \theta\right)$ of the corresponding natural bases are 3-equivalent.

We start with the following simple fact that readily follows from the definitions of the norms.

Fact. Suppose that $\left(\mathcal{F}_{i}, \theta_{i}\right)_{i \in I},\left(\mathcal{G}_{i}, \theta_{i}\right)_{i \in I}$ and $M \subseteq \mathbb{N}$ have the property that every $\mathcal{G}_{i}$-admissible sequence of subsets of $M$ is $\mathcal{F}_{i}$-admissible $(i \in I)$.

Then for every sequence $\left(a_{n}\right)_{n \in M}$ of scalars

$$
\left\|\sum_{n \in M} a_{n} e_{n}\right\|_{\left(\mathcal{G}_{i}, \theta_{i}\right)_{i \in I}} \leq\left\|\sum_{n \in M} a_{n} e_{n}\right\|_{\left(\mathcal{F}_{i}, \theta_{i}\right)_{i \in I}} .
$$

The next is a simple generalization of the above fact that will be used repeatedly.

Proposition 2.1. Suppose that $\left(\mathcal{F}_{i}, \theta_{i}\right)_{i \in I},\left(\mathcal{G}_{i}, \theta_{i}\right)_{i \in I}, M \subseteq \mathbb{N}$ and $k \in \mathbb{N}$ have the property that

$$
[M]^{\leq k} \otimes \operatorname{Ad}\left(\mathcal{F}_{i}\right)\left|M \subseteq \mathcal{G}_{i}\right| M \otimes[M]^{\leq k} \quad(i \in I) .
$$

Then for every sequence $\left(a_{n}\right)_{n \in M}$ of scalars

$$
\left\|\sum_{n \in M} a_{n} e_{n}\right\|_{\left(\mathcal{F}_{i}, \theta_{i}\right)_{i \in I}} \leq k\left\|\sum_{n \in M} a_{n} e_{n}\right\|_{\left(\mathcal{G}_{i}, \theta_{i}\right)_{i \in I}}
$$

Proof. We are going to show, using (3), that for every $\phi \in K^{M}\left(\left(\mathcal{F}_{i}, \theta_{i}\right)_{i \in I}\right)$ there are $\psi_{0}<$ $\cdots<\psi_{l-1}$ in $K^{M}\left(\left(\mathcal{G}_{i}, \theta_{i}\right)_{i \in I}\right), l \leq k$, such that $\phi=\psi_{0}+\cdots+\psi_{l-1}$. The proof is by induction on the complexity of $\phi$ : If $\phi=e_{n}^{*}$, there is nothing to prove. Suppose that $\phi=\theta_{i}\left(\phi_{0}+\cdots+\phi_{n}\right)$, where $\left(\phi_{i}\right)_{i=0}^{n} \subseteq K^{M}\left(\left(\mathcal{F}_{i}, \theta_{i}\right)_{i \in I}\right)$ is $\mathcal{F}_{i}$-admissible. By inductive hypothesis find for every $j$ a set $u_{j}$ of cardinality at most $k$ and a block sequence $\left(\psi_{s}\right)_{s \in u_{j}} \subseteq K^{M}\left(\left(\mathcal{G}_{i}, \theta_{i}\right)_{i \in I}\right)$ such that $\phi_{j}=$ $\sum_{s \in u_{j}} \psi_{s}(j=0, \ldots, n)$. Observe that since $\left(\phi_{j}\right)_{j=0}^{n}$ is $\mathcal{F}_{i}$-admissible, $\left\{\min \phi_{j}\right\}_{j=0}^{n} \in \operatorname{Ad}\left(\mathcal{F}_{i}\right)$. Hence by our hypothesis (3)

$$
t=\bigcup_{j=0}^{n}\left\{\min \psi_{s}: s \in u_{j}\right\} \in[M]^{\leq k} \otimes\left(\operatorname{Ad}\left(\mathcal{F}_{i}\right)\right)\left\lceil M \subseteq \mathcal{G}_{i} \mid M \otimes[M]^{\leq k}\right.
$$


So there are $t_{0}<\cdots<t_{l-1}$ in $\mathcal{G}_{i} \mid M(l \leq k)$ such that $t=t_{0} \cup \cdots \cup t_{l-1}$. For $0 \leq m \leq l-1$ set

$$
\psi^{(m)}=\theta_{i}\left(\sum_{\min \psi_{s} \in t_{m}} \psi_{s}\right) \in K^{M}\left(\left(\mathcal{G}_{i}, \theta_{i}\right)_{i \in I}\right) .
$$

Then $\phi=\psi^{(0)}+\cdots+\psi^{(l-1)}$, as desired.

As a consequence we obtain the following two results. The fist one is the general version of the examples considered in the introduction of this section.

Corollary 2.2. Let $\left(\mathcal{B}_{i}, \theta_{i}\right)_{i=1}^{r}$ and $\left(\mathcal{C}_{i}, \theta_{i}\right)_{i=1}^{r}$ be regular sequences such that $\omega \leq \iota\left(\mathcal{C}_{i}\right) \leq \iota\left(\mathcal{B}_{i}\right) \leq$ $\iota\left(\mathcal{C}_{i}\right) k(1 \leq i \leq r)$ for some integer $k \geq 1$. Then for every $M$ there is some $N \subseteq M$ such that the subsequences $\left(e_{n}\right)_{n \in N}$ of the basis of $T\left[\left(\mathcal{B}_{i}, \theta_{i}\right)_{i=1}^{r}\right]$ and $T\left[\left(\mathcal{C}_{i}, \theta_{i}\right)_{i=1}^{r}\right]$ are $2(k+1)$-equivalent.

Proof. By our assumption on the indexes of the families we obtain that

$$
\iota\left([\mathbb{N}]^{\leq k+1} \otimes \mathcal{B}_{i}\right)=(k+1) \iota\left(\mathcal{B}_{i}\right)<\iota\left(\mathcal{B}_{i}\right)+\omega \leq \iota\left(\mathcal{C}_{i} \otimes[\mathbb{N}] \leq k+1\right)
$$

for every $1 \leq i \leq r$. Hence is some $N_{0} \subseteq M$ such that $\left[N_{0}\right]^{\leq k+1} \otimes \mathcal{B}_{i} \mid N_{0} \subseteq \mathcal{C}_{i} \otimes\left[N_{0}\right]^{\leq k+1}$ for every $1 \leq i \leq r$. Proposition 2.1 yields to

$$
\left\|\sum_{n \in N_{0}} a_{n} e_{n}\right\|_{\left(\mathcal{B}_{i}, \theta_{i}\right)_{i=1}^{r}} \leq(k+1)\left\|\sum_{n \in N_{1}} a_{n} e_{n}\right\|_{\left(\mathcal{C}_{i}, \theta_{i}\right)_{i=1}^{r}}
$$

By Theorem 1.8 there exists $N \subseteq N_{0}$ such that

$$
[N]^{\leq 2} \otimes \mathcal{C}_{i} \mid N \subseteq \mathcal{B}_{i} \otimes[N]^{\leq 2} \text { for every } i \leq r .
$$

Proposition 2.1 yields

$$
\left\|\sum_{n \in N} a_{n} e_{n}\right\|_{\left(\mathcal{C}_{i}, \theta_{i}\right)_{i=1}^{r}} \leq 2\left\|\sum_{n \in N} a_{n} e_{n}\right\|_{\left(\mathcal{B}_{i}, \theta_{i}\right)_{i=1}^{r}}
$$

which completes the proof.

The next result says the shift operator is, when restricted to some subsequence of the basis, always bounded. For a given set $N$ and $n \in N$, let $n^{+} \in N$ be the immediate successor of $n$ in $N$, i.e. $n^{+}=\min N / n$.

Corollary 2.3. Let $\left(\mathcal{B}_{i}, \theta_{i}\right)_{i=1}^{r}$ be a regular sequence. Then for every $M$ there is some $N \subseteq M$ such that for every sequence of scalars $\left(a_{n}\right)_{n \in N}$,

$$
\left\|\sum_{n \in N} a_{n} e_{n}\right\|_{\left.\left(\mathcal{B}_{i}, \theta_{i}\right)_{i=1}^{r}\right)} \leq\left\|\sum_{n \in N} a_{n} e_{n^{+}}\right\|_{\left(\mathcal{B}_{i}, \theta_{i}\right)_{i=1}^{r}} \leq 2\left\|\sum_{n \in N} a_{n} e_{n}\right\|_{\left(\mathcal{B}_{i}, \theta_{i}\right)_{i=1}^{r}} .
$$

Proof. We set $I=\left\{1 \leq i \leq r: \iota\left(\mathcal{B}_{i}\right)\right.$ is finite $\}$ and $J$ for complement of $I$. By Theorem 1.8 we can find $N \subseteq M$ such that and

$$
[N]^{\leq 2} \otimes\left(\left(\mathcal{B}_{i} \mid N\right) \oplus[N]^{\leq 1}\right) \subseteq\left(\mathcal{B}_{i} \mid N\right) \otimes[N]^{\leq 2} \quad(i \in J)
$$

Moreover, we may assume that $\mathcal{B}_{i} \mid N=[N]^{\leq \iota}\left(\mathcal{B}_{i}\right)$ for every $i \in I$ (see Proposition 3.4). By Proposition 2.1 we get

$$
\left\|\sum_{n \in N} a_{n} e_{n}\right\|_{\left(\mathcal{B}_{i}, \theta_{i}\right)_{i \in I}} \frown\left(\mathcal{B}_{i} \oplus[\mathbb{N}] \leq 1, \theta_{i}\right)_{i \in J} \leq 2\left\|\sum_{n \in N} a_{n} e_{n}\right\|_{\left(\mathcal{B}_{i}, \theta_{i}\right)_{i=1}^{r}} .
$$

Observe that for every finite set $s \subseteq N$, setting $s^{+}=\left\{n^{+}: n \in s\right\} \in \mathcal{B}_{i}$, then for $i \in I$ it holds that $s \in \mathcal{F}_{i}$, while for $i \in J, s^{+} \preceq{ }_{*} s$, hence ${ }_{*} s \in \mathcal{B}_{i}\left(\mathcal{B}_{i}\right.$ is spreading) and so $s \in \mathcal{B}_{i} \oplus[\mathbb{N}] \leq 1$. This fact proves that

$$
\left\|\sum_{n \in N} a_{n} e_{n^{+}}\right\|_{\left(\mathcal{B}_{i}, \theta_{i}\right)_{i=1}^{r}} \leq\left\|\sum_{n \in N} a_{n} e_{n}\right\|_{\left(\mathcal{B}_{i}, \theta_{i}\right)_{i \in I}} \frown\left(\mathcal{B}_{i} \oplus[\mathbb{N}] \leq 1, \theta_{i}\right)_{i \in J} .
$$

Now, using that $\mathcal{B}_{i}$ are spreading, by (5) and (6) we get,

$$
\left\|\sum_{n \in N} a_{n} e_{n}\right\|_{\left(\mathcal{B}_{i}, \theta_{i}\right)_{i=1}^{r}} \leq\left\|\sum_{n \in N} a_{n} e_{n+}\right\|_{\left(\mathcal{B}_{i}, \theta_{i}\right)_{i=1}^{r}} \leq 2\left\|\sum_{n \in N} a_{n} e_{n}\right\|_{\left(\mathcal{B}_{i}, \theta_{i}\right)_{i=1}^{r}} .
$$


We examine the effect of the power operation $\mathcal{B}^{\otimes(m)}$ for regular families $\mathcal{B}$ on the corresponding norming set. We follow some of the ideas used in the proof of the corresponding result for Schreier families (see [15], 117]).

Lemma 2.4. Fix an infinite set $M$ of integers, $m \in \mathbb{N}$ and a regular sequence $\left(\mathcal{B}_{i}, \theta_{i}\right)_{i=1}^{r}$. Then for every sequence $\left(a_{n}\right)_{n \in M}$ of scalars

$$
\left\|\sum_{n \in M} a_{n} e_{n}\right\|_{\left(\mathcal{B}_{1}^{\otimes(m)}, \theta_{1}^{m}\right) \frown\left(\mathcal{B}_{i}, \theta_{i}\right)_{i=2}^{r}} \leq\left\|\sum_{n \in M} a_{n} e_{n}\right\|_{\left(\mathcal{B}_{i}, \theta_{i}\right)_{i=1}^{r}} .
$$

Proof. For simplicity, using that the families considered here are regular, we may assume that $M=\mathbb{N}$. Suppose that $\phi \in K\left(\left(\mathcal{B}_{1}^{\otimes(m)}, \theta_{1}^{m}\right)^{\frown}\left(\mathcal{B}_{i}, \theta_{i}\right)_{i=2}^{r}\right)$. We will show that

$$
\phi\left(\sum_{n} a_{n} e_{n}\right) \leq\left\|\sum_{n} a_{n} e_{n}\right\|_{\left(\mathcal{B}_{i}, \theta_{i}\right)_{i=1}^{r}} .
$$

It can be easily shown by induction on $m$ that if $\left(s_{i}\right)_{i=1}^{k}$ is $\mathcal{B}_{1}^{\otimes(m)}$-admissible, then

$$
\theta^{m} \sum_{i=1}^{k}\left\|\sum_{n \in s_{i}} a_{n} e_{n}\right\|_{\left(\mathcal{B}_{i}, \theta_{i}\right)_{i=1}^{r}} \leq\left\|\sum_{n \in \bigcup_{i=1}^{k} s_{i}} a_{n} e_{n}\right\|_{\left(\mathcal{B}_{i}, \theta_{i}\right)_{i=1}^{r}} .
$$

It is not difficult show by induction on the complexity of $\phi$ that the last inequality gives (8).

Lemma 2.5. Suppose that $M$ is an infinite set and that $\left(\mathcal{B}_{i}, \theta_{i}\right)_{i=1}^{r}$ is a regular sequence such that

$$
\mathcal{B}_{1} \uparrow M \otimes \mathcal{B}_{i} \subseteq \mathcal{B}_{i} \otimes \mathcal{B}_{1}
$$

for every $1 \leq i \leq r$. Then for every integer $m$,

$$
\theta_{1}^{m-1}\left\|\sum_{n \in M} a_{n} e_{n}\right\|_{\left(\mathcal{B}_{i}, \theta_{i}\right)_{i=1}^{r}} \leq\left\|\sum_{n \in M} a_{n} e_{n}\right\|_{\left(\mathcal{B}_{1}^{\otimes(m)}, \theta_{1}^{m}\right)-\left(\mathcal{B}_{i}, \theta_{i}\right)_{i=2}^{r}} \leq\left\|\sum_{n \in M} a_{n} e_{n}\right\|_{\left(\mathcal{B}_{i}, \theta_{i}\right)_{i=1}^{r}} .
$$

Proof. The second inequality is given by the previous Lemma 2.4. We assume that $M=\mathbb{N}$. In order to prove the first inequality of (11) we are going to show that

$$
\phi\left(\sum_{n} a_{n} e_{n}\right) \leq \frac{1}{\theta^{m-1}}\left\|\sum_{n} a_{n} e_{n}\right\|_{\left.\left(\mathcal{B}_{1}^{\otimes(m)}, \theta_{1}^{m}\right) \frown\left(\mathcal{B}_{i}, \theta_{i}\right)_{i=2}^{r}\right)}
$$

for every if $\phi \in K\left(\left(\mathcal{B}_{i}, \theta_{i}\right)_{i=1}^{r}\right)$ : For suppose that $\left(\phi_{t}\right)_{t \in \mathcal{T}}$ is a tree analysis of $\phi$. For every $s \preceq t$ and $1 \leq i \leq r$ let

$$
l_{i}(s, t)=\#\left(\left\{u: s \preceq u \supsetneqq t \text { and } \phi_{u}=\theta_{i} \sum_{v \in S_{u}} \phi_{v}\right\}\right) .
$$

So we have the decomposition

$$
\phi=\sum_{t \in \mathcal{A}}\left(\prod_{i=1}^{r} \theta_{i}^{n_{i}(t)}\right)(-1)^{\varepsilon_{t}} e_{m_{t}}
$$

where $\mathcal{A}$ is the set of terminal nodes of $\mathcal{T}, n_{i}(t)=l_{i}(\emptyset, t), \varepsilon_{t} \in\{0,1\}$, and $m_{t}$ is an integer.

Claim. Suppose that there is some $0 \leq d<m$ such that $n_{1}(t) \equiv d(\bmod m)$ for every $t \in \mathcal{A}$. Then there are $\left(\psi_{i}\right)_{i=1}^{l} \subseteq K\left(\left(\mathcal{B}_{1}^{\otimes(m)}, \theta_{1}^{m}\right)^{\frown}\left(\mathcal{B}_{i}, \theta_{i}\right)_{i=2}^{r}\right)$ such that

(a) $\phi=\theta_{1}^{d}\left(\psi_{1}+\cdots+\psi_{l}\right)$

(b) $\left(\psi_{i}\right)_{i=1}^{l}$ is $\mathcal{B}_{1}^{\otimes(d)}$-admissible.

Assuming the Claim, for every $t \in \mathcal{A}$, let $0 \leq d_{t}<m$ be such that $n_{1}(t)+d_{t} \equiv 0(\bmod m)$, and let

$$
\psi=\sum_{t \in \mathcal{A}}\left(\theta_{1}^{d_{t}} \prod_{i=1}^{r} \theta_{i}^{n_{i}(t)}\right)(-1)^{\varepsilon_{t}} e_{m_{t}} .
$$


By the Claim we have that $\psi \in K\left(\left(\mathcal{B}_{1}^{\otimes(m)}, \theta_{1}^{m}\right)^{\frown}\left(\mathcal{B}_{i}, \theta_{i}\right)_{i=2}^{r}\right)$. Finally,

$$
\left|\phi\left(\sum_{n} a_{n} e_{n}\right)\right| \leq \frac{1}{\theta_{1}^{m-1}}\left|\psi\left(\sum_{n} a_{n} e_{n}\right)\right| \leq \frac{1}{\theta^{m-1}}\left\|\sum_{n} a_{n} e_{n}\right\|_{\left(\mathcal{B}_{1}^{\otimes(m)}, \theta_{1}^{m}\right)-\left(\mathcal{B}_{i}, \theta_{i}\right)_{i=2}^{r}} .
$$

which completes the proof of the Lemma.

Proof of Claim: The proof is by induction on the complexity of $\phi$. Suppose first that $\phi= \pm e_{s}$. Then $d=0$ and the desired result is clearly true. Now suppose that $\phi=\theta_{j}\left(\phi_{1}+\cdots+\phi_{k}\right)$. There are two cases to consider. If $j=1$, then, by inductive hypothesis applied to each $\phi_{i}$ $(1 \leq i \leq k)$, we have that for every $1 \leq i \leq k$,

$$
\phi_{i}=\theta_{1}^{\bar{d}}\left(\psi_{1}^{(i)}+\cdots+\psi_{s_{i}}^{(i)}\right)
$$

where $0 \leq \bar{d}<m$ is such that $\bar{d} \equiv d-1(\bmod m),\left(\psi_{l}^{(i)}\right)_{l=1}^{s_{i}} \subseteq K\left(\left(\mathcal{B}_{1}^{\otimes(m)}, \theta_{1}^{m}\right)^{\frown}\left(\mathcal{B}_{i}, \theta_{i}\right)_{i=2}^{r}\right)$ is $\mathcal{B}^{\otimes(\bar{d})}$-admissible. It follows that

$$
\phi=\theta_{1}\left(\phi_{1}+\cdots+\phi_{k}\right)= \begin{cases}\theta_{1}^{m}\left(\sum_{i=1}^{k}\left(\sum_{l=1}^{s_{i}} \psi_{l}^{(i)}\right)\right) & \text { if } d=0 \\ \theta_{1}^{d}\left(\sum_{i=1}^{k}\left(\sum_{l=1}^{s_{i}} \psi_{l}^{(i)}\right)\right) & \text { if } d>0\end{cases}
$$

Using that $\left(\phi_{i}\right)_{i=1}^{k}$ is $\mathcal{B}_{1}$-admissible we obtain that

$$
\bigcup_{i=1}^{k}\left\{\min \psi_{j}^{(i)}\right\}_{j=1}^{s_{i}} \in \begin{cases}\mathcal{B}_{1}^{\otimes(m)} & \text { if } d=0 \\ \mathcal{B}_{1}^{\otimes(d)} & \text { if } d>0 .\end{cases}
$$

So if $d=0$ we obtain that $\phi \in K\left(\left(\mathcal{B}_{1}^{\otimes(m)}, \theta_{1}^{m}\right) \frown\left(\mathcal{B}_{i}, \theta_{i}\right)_{i=2}^{r}\right)$, as desired; otherwise, (a) and (b) in the claim are clearly true for $\phi$.

Now suppose that $j>1$. By inductive hypothesis applied to each $\phi_{i}(1 \leq i \leq k)$, we have that for every $1 \leq i \leq k$,

$$
\phi_{i}=\theta_{1}^{d}\left(\psi_{1}^{(i)}+\cdots+\psi_{s_{i}}^{(i)}\right)
$$

where $\left(\psi_{l}^{(i)}\right)_{l=1}^{k_{i}} \subseteq K\left(\left(\mathcal{B}_{1}^{\otimes(m)}, \theta_{1}^{m}\right) \frown\left(\mathcal{B}_{i}, \theta_{i}\right)_{i=2}^{r}\right)$ is $\mathcal{B}_{1}^{\otimes(d)}$-admissible. It follows that the sequence $\left(\psi_{1}^{(1)}, \ldots, \psi_{s_{1}}^{(1)}, \ldots, \psi_{1}^{(k)}, \ldots, \psi_{s_{k}}^{(k)}\right)$ is $\left(\mathcal{B}_{1}^{\otimes(d)}\right) \otimes \mathcal{B}_{j^{-}}$-admissible. Observe that (10) and the associative property of $\otimes$ give that

$$
\left(\mathcal{B}_{1}^{\otimes(d)}\right) \otimes \mathcal{B}_{j}=\left(\mathcal{B}_{1} \otimes \stackrel{(d)}{\cdots} \otimes \mathcal{B}_{1}\right) \otimes \mathcal{B}_{j} \subseteq \mathcal{B}_{j} \otimes\left(\mathcal{B}_{1}^{\otimes(d)}\right),
$$

so it follows that $\left(\psi_{1}^{(1)}, \ldots, \psi_{s_{1}}^{(1)}, \ldots, \psi_{1}^{(k)}, \ldots, \psi_{s_{k}}^{(k)}\right)$ is also $\mathcal{B}_{j} \otimes\left(\mathcal{B}_{1}^{\otimes(d)}\right)$-admissible. Let $\left(t_{i}\right)_{i=1}^{h}$ be a block sequence of finite sets such that

$$
\left\{\min \psi_{p}^{(i)}: 1 \leq i \leq k, 1 \leq p \leq s_{i}\right\}=\bigcup_{i=1}^{h} t_{i}
$$

with $\left\{t_{i}\right\}_{i=1}^{h} \subseteq \mathcal{B}_{j}$ and $\left\{\min t_{i}\right\}_{i=1}^{h} \in \mathcal{B}_{1}^{\otimes(d)}$. For every $1 \leq l \leq h$ let

$$
\xi_{l}=\theta_{j} \sum_{\min \psi_{p}^{(i)} \in t_{l}} \psi_{p}^{(i)} \in K\left(\left(\mathcal{B}_{1}^{\otimes(m)}, \theta_{1}^{m}\right)^{\frown}\left(\mathcal{B}_{i}, \theta_{i}\right)_{i=2}^{r}\right) .
$$

Whence we obtain the decomposition

$$
\phi=\theta_{1}^{d} \sum_{l=1}^{h} \xi_{l}
$$

giving the desired result.

As a consequence of the previous lemma we get the next proposition which is the natural generalization of a well know fact for the Schreier families $\mathcal{S}_{n}(n \in \mathbb{N})$. 
Proposition 2.6. Let $\mathcal{B}$ be a regular family. Then for every $0<\theta<1$, every $m$, and every sequence of scalars $\left(a_{n}\right)$

$$
\left\|\sum_{n} a_{n} e_{n}\right\|_{\left(\mathcal{B} \otimes(m), \theta^{m}\right)} \leq\left\|\sum_{n} a_{n} e_{n}\right\|_{(\mathcal{B}, \theta)} \leq \frac{1}{\theta^{m-1}}\left\|\sum_{n} a_{n} e_{n}\right\|_{\left(\mathcal{B} \otimes(m), \theta^{m}\right)} .
$$

The next lemma intends to analyze the case of indexes $\iota(\mathcal{B})=\omega^{\alpha+\beta}$ and $\iota(\mathcal{C})=\omega^{\alpha}$ with $\alpha \geq \omega$ and $\beta<\alpha$, for example $\mathcal{C}=\mathcal{S}_{\omega^{2}+\omega}$ and $\mathcal{G}=\mathcal{S}_{\omega^{2}}$.

Lemma 2.7. Let $M$ be an infinite set of integers, $\mathcal{C}, \mathcal{B}_{i}$ be regular families $(1 \leq i \leq r)$ such that $[M]^{\leq 2} \subseteq \mathcal{C}$ and

$$
[M]^{\leq 2} \otimes \mathcal{C}\left|M \otimes \mathcal{B}_{i}\right| M \subseteq \mathcal{B}_{i} \otimes[\mathbb{N}]^{\leq 2} \quad(1 \leq i \leq r)
$$

Then for every sequence $\left(\theta_{i}\right)_{i=1}^{r} \subset(0,1)$ and every sequence of scalars $\left(a_{n}\right)_{n \in M}$,

$$
\left\|\sum_{n \in M} a_{n} e_{n}\right\|_{\left(\mathcal{B}_{i}, \theta_{i}\right)_{i=1}^{r}} \leq\left\|\sum_{n \in M} a_{n} e_{n}\right\|_{\left(\mathcal{B}_{1} \otimes \mathcal{C}, \theta_{1}\right)}-\left(\mathcal{B}_{i}, \theta_{i}\right)_{i=2}^{r} \leq \frac{2}{\theta_{1}}\left\|\sum_{n \in M} a_{n} e_{n}\right\|_{\left(\mathcal{B}_{i}, \theta_{i}\right)_{i=1}^{r}} .
$$

Proof. The first inequality is clear. Let us show the second one. In order to keep the notation simpler, we may assume, since all families here are regular, that $M=\mathbb{N}$.

Claim. Every $\phi \in K\left(\left(\mathcal{B}_{1} \otimes \mathcal{C}, \theta_{1}\right)^{\frown}\left(\mathcal{B}_{i}, \theta_{i}\right)_{i=2}^{r}\right)$ has a decomposition

$$
\phi=\phi_{1}+\cdots+\phi_{n},
$$

where $\left(\phi_{i}\right)_{i=1}^{n} \subseteq K\left(\left(\mathcal{C} \otimes \mathcal{B}_{1}, \theta_{1}\right)^{\frown}\left(\mathcal{B}_{i}, \theta_{i}\right)_{i=2}^{r}\right)$ is $\mathcal{C}$-admissible.

Proof of Claim: Fix $\phi \in K\left(\left(\mathcal{B}_{1} \otimes \mathcal{C}, \theta_{1}\right)^{\frown}\left(\mathcal{B}_{i}, \theta_{i}\right)_{i=2}^{r}\right)$. If $\phi= \pm e_{n}^{*}$, the claim is clear. Now there are two cases to consider.

CASE 1. $\phi=\theta_{1}\left(\phi_{1}+\cdots+\phi_{n}\right)$, where $\left(\phi_{i}\right)_{i=1}^{n} \subseteq K\left(\left(\mathcal{B}_{1} \otimes \mathcal{C}, \theta_{1}\right)^{\frown}\left(\mathcal{B}_{i}, \theta_{i}\right)_{i=2}^{r}\right)$ is $\mathcal{B}_{1} \otimes \mathcal{C}$-admissible. By inductive hypothesis, for each $i=1, \ldots, n$,

$$
\phi_{i}=\sum_{j=1}^{n_{i}} \psi_{j}^{(i)}
$$

where $\left(\psi_{j}^{(i)}\right)_{j=1}^{n_{i}} \subseteq K\left(\left(\mathcal{C} \otimes \mathcal{B}_{1}, \theta_{1}\right)^{\frown}\left(\mathcal{B}_{i}, \theta_{i}\right)_{i=2}^{r}\right)$ is $\mathcal{C}$-admissible, i.e. $s_{i}=\left\{\min \psi_{j}^{(i)}\right\}_{j=1}^{n_{i}} \in \mathcal{C}$. Since for every $i=1, \ldots, n, \min s_{i}=\min \operatorname{supp} \phi_{i}$ we obtain, by (26), that

$$
s_{1} \cup \cdots \cup s_{n} \in \mathcal{C} \otimes\left(\mathcal{B}_{1} \otimes \mathcal{C}\right)=\left(\mathcal{C} \otimes \mathcal{B}_{1}\right) \otimes \mathcal{C} .
$$

Hence we can find a block sequence $\left(t_{i}\right)_{i=1}^{m}$ such that

$$
s_{1} \cup \cdots \cup s_{n}=t_{1} \cup \cdots \cup t_{m}
$$

and such that $\left(t_{i}\right)_{i=1}^{m} \subseteq \mathcal{C} \otimes \mathcal{B}_{1}$ is $\mathcal{C}$-admissible. For every $k \in t_{1} \cup \cdots \cup t_{m}$, let $i(k), j(k)$ be such that

$$
\min \psi_{j(k)}^{(i(k))}=k
$$

For every $i=1, \ldots, m$, let

$$
\psi_{i}=\theta_{1}\left(\sum_{k \in t_{i}} \psi_{j(k)}^{i(k)}\right) .
$$

Since $\left(\psi_{j(k)}^{(i(k))}\right)_{k \in t_{i}}$ is a block sequence, and since $\left\{\min \psi_{j(k)}^{(i(k))}\right\}_{k \in t_{i}}=t_{i} \in \mathcal{C} \otimes \mathcal{B}_{1}$ we obtain that $\psi_{i} \in K\left(\left(\mathcal{C} \otimes \mathcal{B}_{1}, \theta_{1}\right)^{\frown}\left(\mathcal{B}_{i}, \theta_{i}\right)_{i=2}^{r}\right)$. It is clear that

$\phi=\theta_{1}\left(\phi_{1}+\cdots+\phi_{n}\right)=\theta_{1}\left(\sum_{i=1}^{n} \sum_{j=1}^{n_{i}} \psi_{j}^{(i)}\right)=\theta_{1} \sum_{i=1}^{m} \sum_{k \in t_{i}} \psi_{j(k)}^{(i(k))}=\sum_{i=1}^{m} \theta_{1} \sum_{k \in t_{i}} \psi_{j(k)}^{(i(k))}=\psi_{1}+\cdots+\psi_{m}$.

Note that $\min \psi_{i}=\min t_{i}(1 \leq i \leq m)$, hence $\left\{\min \psi_{i}\right\}_{i=1}^{m}=\left\{\min t_{i}\right\}_{i=1}^{m} \in \mathcal{C}$, so we are done. 
CASE 2. $\phi=\theta_{j}\left(\phi_{1}+\cdots+\phi_{n}\right)$, where $\left(\phi_{i}\right)_{i=1}^{n} \subseteq K\left(\left(\mathcal{B}_{1} \otimes \mathcal{C}, \theta_{1}\right)^{\frown}\left(\mathcal{B}_{i}, \theta_{i}\right)_{i=2}^{r}\right)$ is $\mathcal{B}_{j}$-admissible for some $2 \leq j \leq r$. By inductive hypothesis, for each $i=1, \ldots, n$,

$$
\phi_{i}=\sum_{j=1}^{n_{i}} \psi_{j}^{(i)}
$$

where $\left.\left(\psi_{j}^{(i)}\right)_{j=1}^{n_{i}} \subseteq K\left(\left(\mathcal{C} \otimes \mathcal{B}_{1}, \theta_{1}\right)^{\frown}\left(\mathcal{B}_{i}, \theta_{i}\right)_{i=2}^{r}\right)\right)$ is $\mathcal{C}$-admissible, i.e. $s_{i}=\left\{\min \psi_{j}^{(i)}\right\}_{j=1}^{n_{i}} \in \mathcal{C}$. It follows, by $(26)$ and the fact that $[\mathbb{N}] \leq 2 \subseteq \mathcal{C}$, that

$$
s_{1} \cup \cdots \cup s_{n} \in \mathcal{C} \otimes \mathcal{B}_{j} \subseteq \mathcal{B}_{j} \otimes[\mathbb{N}]^{\leq 2} \subseteq \mathcal{B}_{j} \otimes \mathcal{C} .
$$

Following similar ideas than in the proof of the Case 1 one can easily find the desired decomposition of $\phi$.

From the claim we obtain that $\theta_{1} \phi \in K\left(\left(\mathcal{C} \otimes \mathcal{B}_{1}\right)^{\frown}\left(\mathcal{B}_{i}, \theta_{i}\right)_{i=2}^{r}\right)$ for every $\phi \in K\left(\left(\mathcal{B}_{1} \otimes\right.\right.$ $\left.\left.\mathcal{C}, \theta_{1}\right)^{\frown}\left(\mathcal{B}_{i}, \theta_{i}\right)_{i=2}^{r}\right)$. Now this fact implies that for every sequence $\left(a_{n}\right)_{n}$ of scalars

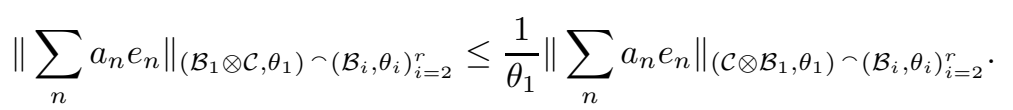

Since (26) holds, we can apply Proposition 2.1 to get that

$$
\left\|\sum_{n} a_{n} e_{n}\right\|_{\left(\mathcal{C} \otimes \mathcal{B}_{1}, \theta_{1}\right)}-\left(\mathcal{B}_{i}, \theta_{i}\right)_{i=2}^{r} \leq 2\left\|\sum_{n} a_{n} e_{n}\right\|_{\left(\mathcal{B}_{i}, \theta_{i}\right)_{i=1}^{r}} .
$$

Finally we obtain the desired inequality by joining (27) and (28).

Theorem 2.8. Suppose that $\mathcal{B}_{0}$ and $\mathcal{B}_{1}$ are two regular families such that $\iota\left(\mathcal{B}_{0}\right)=\omega^{\alpha+\beta}$, $\iota\left(\mathcal{B}_{1}\right)=\omega^{\alpha}$, with $0<\beta<\alpha$, and $\alpha \geq \omega$. Then for every infinite set $M$ of integers there is an infinite $N \subseteq M$ such that $\left(e_{n}\right)_{n \in N} \subseteq T\left(\mathcal{B}_{0}, \theta\right)$ and $\left(e_{n}\right)_{n \in N} \subseteq T\left(\mathcal{B}_{1}, \theta\right)$ are equivalents.

Proof. Let $\mathcal{C}$ be a regular family with $\iota(\mathcal{C})=\omega^{\beta}$. Since $\iota\left(\mathcal{B}_{1} \otimes \mathcal{C}\right)=\omega^{\alpha+\beta}=\iota\left(\mathcal{B}_{0}\right)$ passing to a subset $N$ of $M$, we may assume that the subsequence $\left(e_{n}\right)_{n \in N}$ is equivalent in the spaces $T\left(\mathcal{B}_{0}, \theta_{0}\right)$ and $T\left(\mathcal{B}_{1} \otimes \mathcal{C}, \theta_{0}\right)$, and hence we may assume that $\mathcal{B}_{0}=\mathcal{B}_{1} \otimes \mathcal{C}$. The result follows from the previous lemma.

2.1. Reduction from finite to one. The aim of this subsection is to reduce finite regular sequences to one, more precisely, we show in Theorem 2.13 that for every finite regular sequence $\left(\mathcal{F}_{i}, \theta_{i}\right)_{i=1}^{r}$ there is some $1 \leq i_{0} \leq r$ and some infinite set $M$ of integers such that $\left(e_{n}\right)_{n \in M} \subseteq T\left[\left(\mathcal{F}_{i}, \theta_{i}\right)_{i=1}^{r}\right]$ and $\left(e_{n}\right)_{\in M} \subseteq T\left(\mathcal{F}_{i_{0}}, \theta_{i_{0}}\right)$ are equivalent, where $i_{0}$ will come from a certain ordering of the pairs $\left(\mathcal{F}_{i}, \theta_{i}\right)$.

Definition 2.9. Recall that every ordinal $\alpha>0$ has the unique decomposition

$$
\alpha=\omega^{\lambda(\alpha)} k(\alpha)+\xi(\alpha)
$$

with $k(\alpha)$ an integer and $\xi(\alpha)<\omega^{\lambda(\alpha)}$. Define

$$
\begin{aligned}
& \gamma(\alpha)= \begin{cases}k(\alpha) & \text { if } \alpha \text { is finite } \\
\omega^{\omega^{\lambda(\lambda(\alpha))}} & \text { if } \alpha \text { is infinite },\end{cases} \\
& n(\alpha)= \begin{cases}1 & \text { if } \alpha \text { is finite } \\
k(\lambda(\alpha)) & \text { if } \alpha \text { is infinite. }\end{cases}
\end{aligned}
$$

For example, $\gamma\left(\omega^{\omega^{2} 3+\omega} 4+\omega^{5}\right)=\omega^{\omega^{2}}, n\left(\omega^{\omega^{2} 3+\omega} 4+\omega^{5}\right)=3$ and $\gamma(m)=m$ for every integer $m$. In general for an arbitrary ordinal $\alpha$ we have the decomposition

$$
\alpha=\gamma(\alpha)^{n(\alpha)} \omega^{\xi(\lambda(\alpha))} k(\alpha)+\xi(\alpha),
$$

with the convention of $\xi(0)=0$. 
We want to compare two Tsirelson type spaces $T\left(\mathcal{F}_{0}, \theta_{0}\right)$ and $T\left(\mathcal{F}_{1}, \theta_{1}\right)$. There is the following natural relation of domination: we write $\left(\mathcal{F}_{0}, \theta_{0}\right) \leq^{\prime}\left(\mathcal{F}_{1}, \theta_{1}\right)$ iff there is some $C \geq 1$ such that every subsequence $\left(e_{n}\right)_{n \in M}$ of the basis of $T\left(\mathcal{F}_{0}, \theta_{0}\right)$ has a further subsequence $\left(e_{n}\right)_{n \in N}$ such that

$$
\left\|\sum_{n \in N} a_{n} e_{n}\right\|_{\left(\mathcal{F}_{0}, \theta_{0}\right)} \leq C\left\|\sum_{n \in N} a_{n} e_{n}\right\|_{\left(\mathcal{F}_{1}, \theta_{1}\right)}
$$

It is clear that if $\mathcal{F}_{0} \subseteq \mathcal{F}_{1}$ and $\theta_{0} \leq \theta_{1}$ the pair $\left(\mathcal{F}_{1}, \theta_{1}\right)$ dominates $\left(\mathcal{F}_{0}, \theta_{0}\right)$. As we have already seen in Proposition 2.6 the pairs $(\mathcal{F}, \theta)$ and $\left(\mathcal{F}^{\otimes(n)}, \theta_{n}\right)$ are mutually dominated $(n \in \mathbb{N})$. This suggests the following more appropriate relation: $\left(\mathcal{F}_{0}, \theta_{0}\right) \leq^{\prime \prime}\left(\mathcal{F}_{1}, \theta_{1}\right)$ iff there are $n_{0}, n_{1} \in \mathbb{N}$ such that for every $M$ there is $N \subseteq M$ such that $\mathcal{F}_{0}^{\otimes\left(n_{0}\right)}\left\lceil N \subseteq \mathcal{F}_{1}^{\otimes\left(n_{1}\right)}\right.$ and $\theta_{0}^{n_{0}} \leq \theta_{1}^{n_{1}}$.

We have also shown that $\left(\mathcal{S}_{\omega^{\alpha}+\beta} \otimes[N]^{\leq k}, \theta\right),\left(\mathcal{S}_{\omega^{\alpha}+\beta}, \theta\right)$ and $\left(\mathcal{S}_{\omega^{\alpha}}, \theta\right)$ are all of them mutually dominated, that leads to the following definition:

Definition 2.10. For pairs $(\alpha, \theta)$ of ordinals and real numbers we write $\left(\alpha_{0}, \theta_{0}\right) \leq_{\mathrm{T}}\left(\alpha_{1}, \theta_{1}\right)$ iff (1) $\alpha_{0} \alpha_{1}<\omega$ and $\log _{\gamma\left(\alpha_{0}\right)} \theta_{0} \leq \log _{\gamma\left(\alpha_{1}\right)} \theta_{1}$, or

(2) $\alpha_{0} \alpha_{1} \geq \omega$ and there are integers $m_{0}, m_{1}$ such that $\gamma\left(\alpha_{0}\right)^{n\left(\alpha_{0}\right) m_{0}} \leq \gamma\left(\alpha_{1}\right)^{n\left(\alpha_{0}\right) m_{1}}$ and $\theta_{0}^{m_{0}} \leq$ $\theta_{1}^{m_{1}}$.

We write $\left(\mathcal{F}_{0}, \theta_{0}\right) \leq_{\mathrm{T}}\left(\mathcal{F}_{1}, \theta_{1}\right)$ iff $\left(\iota\left(\mathcal{F}_{0}\right), \theta_{0}\right) \leq_{\mathrm{T}}\left(\iota\left(\mathcal{F}_{1}\right), \theta_{1}\right)$.

To keep the notation easier we will write $\gamma(\mathcal{F})$ for $\gamma(\iota(\mathcal{F}))$ and $n(\mathcal{F})$ for $n(\iota(\mathcal{F}))$. Few more properties:

Proposition 2.11. (a) Suppose that $\max \left\{\alpha_{0}, \alpha_{1}\right\} \geq \omega$. Then $\left(\alpha_{0}, \theta_{0}\right) \leq_{\mathrm{T}}\left(\alpha_{1}, \theta_{1}\right)$ iff $\gamma\left(\alpha_{0}\right)<$ $\gamma\left(\alpha_{1}\right)$, or if $\gamma\left(\alpha_{0}\right)=\gamma\left(\alpha_{1}\right)$ then $\theta_{0}^{n\left(\alpha_{1}\right)} \leq \theta_{1}^{n\left(\alpha_{0}\right)}$.

(b) $<_{\mathrm{T}}$ is a total ordering.

Proof. (b): We show that $<_{\mathrm{T}}$ is total. So, fix two pairs $\left(\alpha_{i}, \theta_{i}\right)(i=0,1)$. Suppose first that $\alpha_{i} \omega \leq \alpha_{j}$ for $i \neq j$. Then let $n$ be such that $\theta_{i}^{n}<\theta_{j}$. Then clearly $\alpha_{i} n<\alpha_{j}$, and $\theta_{i}^{n}<\theta_{j}$, so $\left(\alpha_{i}, \theta_{i}\right)<_{\mathrm{T}}\left(\alpha_{j}, \theta_{j}\right)$. Suppose now that $\gamma\left(\alpha_{0}\right)=\gamma\left(\alpha_{1}\right)$. Then if $\theta_{0}^{n\left(\alpha_{1}\right)} \leq \theta_{1}^{n\left(\alpha_{0}\right)}$ we obtain that $\left(\alpha_{0}, \theta_{0}\right) \leq_{\mathrm{T}}\left(\alpha_{1}, \theta_{1}\right)$, and $\left(\alpha_{1}, \theta_{1}\right) \leq_{\mathrm{T}}\left(\alpha_{0}, \theta_{0}\right)$ otherwise.

Lemma 2.12. Suppose that $\Gamma$ is a finite set of countable ordinals and $n \in \mathbb{N}$. There is a sequence $\left(\mathcal{B}_{\gamma}\right)_{\gamma \in \Gamma}$ of regular families such that:

(a) $\iota\left(\mathcal{B}_{\gamma}\right)=\gamma$ for every $\gamma \in \Gamma$.

(b) $\mathcal{B}_{\gamma}=[\mathbb{N}] \leq \gamma$ if $\gamma \in \Gamma$ is finite.

(c) For every $m_{1}, m_{2} \leq n$ and every $f_{i}:\left\{1, \ldots, m_{i}\right\} \rightarrow \Gamma(i=1,2)$,

$$
\text { if } \prod_{i \leq m_{1}} f_{1}(i)<\prod_{i \leq m_{2}} f_{2}(i) \text {, then } \mathcal{B}_{f_{1}(1)} \otimes \cdots \otimes \mathcal{B}_{f_{1}\left(m_{1}\right)} \subseteq \mathcal{B}_{f_{2}(1)} \otimes \cdots \otimes \mathcal{B}_{f_{2}\left(m_{2}\right)} .
$$

Proof. Fix for every $\gamma \in \Gamma$ a regular family $\mathcal{C}_{\gamma}$ of index $\gamma$, with the extra requirement that if $\gamma$ is finite then $\mathcal{C}_{\gamma}=[\mathbb{N}] \leq \gamma$. Since $\left\{\mathcal{C}_{f(1)} \otimes \cdots \otimes \mathcal{C}_{f(m)}: f:\{1, \ldots, m\} \rightarrow \Gamma, m \leq n\right\}$ is a finite set of regular families, we can find an infinite set $M$ such that for every $m_{1}, m_{2} \leq n$ and every $f_{i}:\left\{1, \ldots, m_{i}\right\} \rightarrow \Gamma(i=1,2)$, if $\prod_{i \leq m_{1}} f_{1}(i)<\prod_{i \leq m_{2}} f_{2}(i)$, then $\mathcal{C}_{f_{1}(1)} \backslash M \otimes \cdots \otimes \mathcal{C}_{f_{1}\left(m_{1}\right)} \uparrow$ $M \subseteq \mathcal{C}_{f_{2}(1)}\left\lceil M \otimes \cdots \otimes \mathcal{C}_{f_{2}\left(m_{2}\right)}\lceil M\right.$. Let $\bar{\Theta}: M \rightarrow \mathbb{N}$ be the unique order-preserving onto mapping between $M$ and $\mathbb{N}$. Then $\left(\Theta "\left(\mathcal{C}_{\gamma} \mid M\right)\right)_{\gamma \in \Gamma}$ is the desired sequence.

Theorem 2.13. Suppose that $\left(\mathcal{B}_{i}, \theta_{i}\right)_{i=1}^{r}$ is a regular sequence with at least one of the families with infinite index. Let $i_{0}$ be such that $\left(\iota\left(\mathcal{B}_{i_{0}}\right), \theta_{i_{0}}\right)=\max _{<_{\mathrm{T}}}\left\{\left(\iota\left(\mathcal{B}_{i}\right), \theta_{i}\right): 1 \leq i \leq r\right\}$. Then every subsequence $\left(e_{n}\right)_{n \in M}$ of the natural basis of $\left.T\left[\left(\mathcal{B}_{i}, \theta_{i}\right)_{i=1}^{r}\right)\right]$ has a further subsequence $\left(e_{n}\right)_{n \in N}$ equivalent to the corresponding subsequence $\left(e_{n}\right)_{n \in N}$ of the natural basis of $T\left(\mathcal{B}_{i_{0}}, \theta_{i_{0}}\right)$.

Proof. To simplify the notation we assume that $M=\mathbb{N}$. We re-order $\left(\mathcal{B}_{i}, \theta_{i}\right)_{i=1}^{r}$ in such a way that $\left(\mathcal{B}_{i}, \theta_{i}\right) \leq_{\mathrm{T}}\left(\mathcal{B}_{j}, \theta_{j}\right)$ for every $1 \leq i \leq j \leq r$. 
Recall the decomposition (see definition 2.9)

$$
\iota\left(\mathcal{B}_{i}\right)=\gamma_{i}^{n_{i}} \delta_{i} k_{i}+\xi_{i}
$$

where if $\iota\left(\mathcal{B}_{i}\right)$ is finite then $\gamma_{i}=\gamma\left(\iota\left(\mathcal{B}_{i}\right)\right), \delta_{i}=n_{i}=k_{i}=1$ and $\xi_{i}=0$, and if $\iota\left(\mathcal{B}_{i}\right)$ is infinite then $n_{i}=n\left(\iota\left(\mathcal{B}_{i}\right)\right), \delta_{i}=\omega^{\xi\left(\lambda\left(\iota\left(\mathcal{B}_{i}\right)\right)\right)}, k_{i}=k\left(\iota\left(\mathcal{B}_{i}\right)\right)$ and $\xi=\xi\left(\iota\left(\mathcal{B}_{i}\right)\right)$. Observe that $\gamma_{r}=\max \left\{\gamma_{i}: 1 \leq i \leq r\right\}$ is infinite. Define $m_{i} \in \mathbb{N}(1 \leq i \leq r-1)$ as

$$
m_{i}= \begin{cases}{\left[\log _{\theta_{i}} \theta_{r}\right]+1} & \text { if } \gamma_{i}<\gamma_{r} \\ n_{r} & \text { if } \gamma_{i}=\gamma_{r}\end{cases}
$$

where $[a]$ stays for the entire part of $a$. Use the previous Lemma 2.12 for $\Gamma=\left\{\gamma_{i}, \delta_{i}\right.$ : $1 \leq i \leq r\} \cup\{2\}$ and $n$ large enough (for example $n=2 \max \left\{n_{i} m_{i}: 1 \leq i \leq r\right\}+2$ ) to find the corresponding sequence $\left(\mathcal{H}_{\gamma}\right)_{\gamma \in \Gamma}$ of regular families.

For $1 \leq i \leq r$, let

$$
\mathcal{C}_{i}=\left(\mathcal{H}_{\gamma_{i}}\right)^{\otimes\left(n_{i}\right)} \otimes \mathcal{H}_{\delta_{i}}
$$

Observe that $\iota\left(\mathcal{C}_{i}\right)=\gamma_{i}^{n_{i}} \omega^{\delta_{i}}$ for every $1 \leq i \leq r$. It readily follows that there is $N \subseteq M$ such that for every $1 \leq i \leq r$, if $\iota\left(\mathcal{B}_{i}\right)$ is infinite then

$$
\begin{array}{r}
{[N]^{\leq 2} \otimes \mathcal{C}_{i} \mid N \subseteq \mathcal{B}_{i} \otimes[N]^{\leq 2}, \text { and }} \\
{[N]^{\leq k_{i}+1} \otimes \mathcal{B}_{i}\left|N \subseteq \mathcal{C}_{i}\right| N \otimes[N]^{\leq k_{i}+1}}
\end{array}
$$

while

$$
\mathcal{B}_{i} \uparrow N=\mathcal{C}_{i} \uparrow N
$$

if $\iota\left(\mathcal{B}_{i}\right)$ is finite. Since the families $\mathcal{B}_{i}$ and $\mathcal{C}_{i}$ are regular $(1 \leq i \leq r)$, Proposition 2.1 gives that for every sequence of scalars $\left(a_{n}\right)_{n \in N}$ we have that

$$
\frac{1}{2}\left\|\sum_{n \in N} a_{n} e_{n}\right\|_{\left(\mathcal{C}_{i}, \theta_{i}\right)_{i=1}^{r}} \leq\left\|\sum_{n \in N} a_{n} e_{n}\right\|_{\left(\mathcal{B}_{i}, \theta_{i}\right)_{i=1}^{r}} \leq\left(1+\max _{1 \leq i \leq r, \iota\left(\mathcal{B}_{i}\right) \text { infinite }} k_{i}\right)\left\|\sum_{n \in N} a_{n} e_{n}\right\|_{\left(\mathcal{C}_{i}, \theta_{i}\right)_{i=1}^{r}}
$$

Let $\left\{\varrho_{i}\right\}_{i=1}^{s}$ be the strictly increasing enumeration of the set $\left\{\gamma_{i}: 1 \leq i \leq r, \gamma_{i}\right.$ infinite $\}$. Define

$$
\begin{aligned}
& I_{0}=\left\{1 \leq i \leq r: \gamma_{i} \text { is finite }\right\} \\
& I_{i}=\left\{1 \leq j \leq r-1: \gamma_{j}=\varrho_{i}\right\} \quad(1 \leq i \leq s),
\end{aligned}
$$

and $I_{s+1}=\{r\}$.

Finally, set $J_{i}=I_{i} \cup \cdots \cup I_{s+1}(0 \leq i \leq s+1)$. The next result is the reduction from $\left(\mathcal{C}_{i}, \theta_{i}\right)_{i=1}^{r}$ to $\left(\mathcal{C}_{r}, \theta_{r}\right)$.

Claim. For every $0 \leq j \leq s$ and every sequence of scalars $\left(a_{n}\right)$ we have that

$$
\left\|\sum_{n} a_{n} e_{n}\right\|_{\left(\mathcal{C}_{i}, \theta_{i}\right)_{i \in J_{j}}} \leq \prod_{i \in I_{j}} \frac{1}{\theta_{i}^{m_{i}-1}} \prod_{i \in I_{j}, \delta_{i}>1} \frac{2}{\theta_{i}}\left\|\sum_{n} a_{n} e_{n}\right\|_{\left(\mathcal{C}_{i}, \theta_{i}\right)_{i \in J_{j+1}}} .
$$

Proof of Claim: Fix $0 \leq j \leq s$. Let $K_{j}=\left\{i \in I_{j}: \delta_{i}>1\right\}$, and suppose it is non-empty. This implies, in particular, that $j>0$. Notice that $\varrho_{j}=\min \left\{\gamma_{k}: k \in J_{j}\right\}$. So it follows that $\delta_{k}<\gamma_{k}=\varrho_{j} \leq \gamma_{i}$ for $k \in K_{j}$ and $i \in J_{j}$. So,

$$
\begin{aligned}
2 \delta_{k} \gamma_{i}^{n_{i}} \delta_{i} & =\gamma_{i}^{n_{i}} \delta_{i}<\gamma_{i}^{n_{i}} \delta_{i} 2 \quad\left(k \in K_{j}, i \in J_{j}\right) \\
2 \delta_{k} \gamma_{i}^{n_{i}} & =\gamma_{i}^{n_{i}}<\gamma_{i}^{n_{i}} 2 \quad\left(i, k \in K_{j}\right) .
\end{aligned}
$$

Hence,

$$
\begin{aligned}
{[\mathbb{N}]^{\leq 2} \otimes \mathcal{H}_{\delta_{k}} \otimes \mathcal{C}_{i} \subseteq \mathcal{C}_{i} \otimes[\mathbb{N}]^{\leq 2} \quad\left(k \in K_{j}, i \in J_{j}\right) } \\
{[\mathbb{N}]^{\leq 2} \otimes \mathcal{H}_{\delta_{k}} \otimes \mathcal{H}_{\gamma_{i}}^{\otimes\left(n_{i}\right)} \subseteq \mathcal{H}_{\gamma_{i}}^{\otimes\left(n_{i}\right)} \otimes[\mathbb{N}]^{\leq 2} \quad\left(i, k \in K_{j}\right) . }
\end{aligned}
$$

A repeated application of Lemma 2.7 gives that

$$
\left\|\sum_{n} a_{n} e_{n}\right\|_{\left(\mathcal{C}_{i}, \theta_{i}\right)_{i \in J_{j}}} \leq \prod_{i \in K_{j}} \frac{2}{\theta_{i}}\left\|\sum_{n} a_{n} e_{n}\right\|_{\left(\mathcal{H}_{\gamma_{i}}^{\otimes\left(n_{i}\right)}, \theta_{i}\right)_{i \in K_{j}}} \frown\left(\mathcal{C}_{i}, \theta_{i}\right)_{i \in J_{j} \backslash K_{j}} .
$$


Using that

it follows that

$$
\gamma_{k}^{n_{k}} \gamma_{i}^{n_{i}} \delta_{i}=\gamma_{i}^{n_{i}} \delta_{i}<\gamma_{i}^{n_{i}} \delta_{i} \gamma_{k}^{n_{k}} \quad\left(k \in I_{j}, i \in J_{j+1}\right)
$$

$$
\mathcal{H}_{\gamma_{k}}^{\otimes\left(n_{k}\right)} \otimes \mathcal{C}_{i} \subseteq \mathcal{C}_{i} \otimes \mathcal{H}_{\gamma_{k}}^{\otimes\left(n_{k}\right)} \quad\left(k \in I_{j}, i \in J_{j+1}\right)
$$

Since it is trivial that $\mathcal{H}_{\varrho_{j}}^{\otimes\left(n_{k}\right)} \otimes \mathcal{H}_{\varrho_{j}}^{\otimes\left(n_{i}\right)}=\mathcal{H}_{\varrho_{j}}^{\otimes\left(n_{k}+n_{i}\right)}=\mathcal{H}_{\varrho_{j}}^{\otimes\left(n_{i}\right)} \otimes \mathcal{H}_{\varrho_{j}}^{\otimes\left(n_{k}\right)}\left(i, k \in I_{j}\right)$, the assumptions of Lemma 2.5 are fulfilled, therefore

$$
\begin{aligned}
\left\|\sum_{n} a_{n} e_{n}\right\|_{\left(\mathcal{H}_{\gamma_{i}}^{\otimes\left(n_{i}\right)}, \theta_{i}\right)_{i \in K_{j}} \frown\left(\mathcal{C}_{i}, \theta_{i}\right)_{i \in J_{j} \backslash K_{j}}}= \\
\quad=\left\|\sum_{n} a_{n} e_{n}\right\|_{\left(\mathcal{H}_{\varrho_{j}}^{\otimes\left(n_{i}\right)}, \theta_{i}\right)_{i \in I_{j}} \frown\left(\mathcal{C}_{i}, \theta_{i}\right)_{i \in J_{j+1}}} \leq \\
\leq \prod_{i \in I_{j}} \frac{1}{\theta_{i}^{m_{i}-1}}\left\|\sum_{n} a_{n} e_{n}\right\|_{\left(\mathcal{H}_{\varrho_{j}}^{\otimes\left(n_{i} m_{i}\right)}, \theta_{i}^{m_{i}}\right)_{i \in I_{j}} \frown\left(\mathcal{C}_{i}, \theta_{i}\right)_{i \in J_{j+1}}} .
\end{aligned}
$$

It is not difficult to see, by the choice of $m_{i}$ 's, that the relations

$$
\begin{array}{r}
\mathcal{H}_{\varrho_{j}}^{\otimes\left(n_{i} m_{i}\right)} \subseteq \mathcal{C}_{r} \text { while } \theta^{m_{i}} \leq \theta_{r}\left(i \in I_{j}\right) \text { if } j<s \text { or } \\
\mathcal{H}_{\varrho_{s}}^{\otimes\left(n_{i} m_{i}\right)}=\mathcal{H}_{\varrho_{s}}^{\otimes\left(n_{i} n_{r}\right)} \subseteq \mathcal{C}_{r}^{\otimes\left(n_{i}\right)} \text { and } \theta_{i}^{m_{i}}=\theta_{i}^{n_{r}} \leq \theta_{r}^{n_{i}}\left(i \in I_{j}\right) \text { if } j=s
\end{array}
$$

are true. Hence, by Lemma 2.4 in the case of $j=s$, we obtain that

$$
\left\|\sum_{n} a_{n} e_{n}\right\|_{\left(\mathcal{H}_{e_{j}}^{\otimes\left(n_{i} m_{i}\right)}, \theta_{i}^{m_{i}}\right)_{i \in I_{j}}} \frown\left(\mathcal{C}_{i}, \theta_{i}\right)_{i \in J_{j+1}} \leq\left\|\sum_{n} a_{n} e_{n}\right\|_{\left(\mathcal{C}_{i}, \theta_{i}\right)_{i \in J_{j+1}}} .
$$

It is clear now that (34) follows from equations (35), (36) and (37).

A repeated application of previous claim gives that

$$
\left\|\sum_{n} a_{n} e_{n}\right\|_{\left(\mathcal{C}_{i}, \theta_{i}\right)_{i=1}^{r}} \leq \prod_{i=1}^{r-1} \frac{1}{\theta_{i}^{m_{i}-1}} \prod_{i=1, \delta_{i}>1}^{r-1} \frac{2}{\theta_{i}}\left\|\sum_{n} a_{n} e_{n}\right\|_{\left(\mathcal{C}_{r}, \theta_{r}\right)} .
$$

It follows from (32), (33) and (38) that

$$
\left\|\sum_{n \in N} a_{n} e_{n}\right\|_{\left(\mathcal{B}_{i}, \theta_{i}\right)_{i=1}^{r}} \leq 2\left(1+\max _{1 \leq i \leq r, \iota\left(\mathcal{B}_{i}\right)} k_{\text {infinite }}\right) \prod_{i=1}^{r-1} \frac{1}{\theta_{i}^{m_{i}-1}} \prod_{i=1, \delta_{i}>1}^{r-1} \frac{2}{\theta_{i}}\left\|\sum_{n \in N} a_{n} e_{n}\right\|_{\left(\mathcal{B}_{r}, \theta_{r}\right)}
$$

In Theorem 2.13 we made the assumption that at least one family $\mathcal{B}_{i}$ has infinite index $(1 \leq i \leq r)$. The conclusion of this theorem is also true for families all of them with finite indexes but its proof uses different methods (see [9]).

\section{Topological And Combinatorial aspects of FAmilies of Finite SETS of INTEgers}

The main result of this section is that for every compact and hereditary family $\mathcal{F}$ there is a regular family $\mathcal{B}$ with the same index than $\mathcal{F}$ and an infinite set $M$ of integers such that every $\mathcal{B}$-admissible sequence of subsets of $M$ is also $\mathcal{F}$-admissible. The main tool we use is the notion of homogeneous family.

We start with the following list of useful properties. We leave their proofs to the reader.

Proposition 3.1. Fix a compact family $\mathcal{F}$, and a countable ordinal $\alpha$. Then

(1) For every $m \in \mathbb{N},\left(\partial^{(\alpha)} \mathcal{F}\right)\left\lceil\mathbb{N} / m=\partial^{(\alpha)}(\mathcal{F} \mid \mathbb{N} / m)\right.$.

(2) $\emptyset \neq s \in \partial^{(\alpha)} \mathcal{F}$ iff ${ }_{*} s \in \partial^{(\alpha)}\left(\mathcal{F}_{\{\min s\}}\right)$.

(3) For every $n \in \mathbb{N}, \partial^{(\alpha)}\left(\mathcal{F}_{\{n\}}\right)=\left(\partial^{(\alpha)} \mathcal{F}\right)_{\{n\}}$.

(4) $\iota(\mathcal{F})=\alpha$ iff (4.1) $\partial^{(\alpha)}\left(\mathcal{F}_{\{n\}}\right)=\emptyset$ for every $n$, and (4.2) for every $\beta<\alpha$ there is some $n$ such that $\partial^{(\beta)} \mathcal{F}_{\{n\}} \neq \emptyset$.

$(5) \iota(\mathcal{F})=\alpha$ limit implies that $\partial^{(\alpha)} \mathcal{F}=\{\emptyset\}$ and for every $n \in \mathbb{N}, \iota(\mathcal{F} \uparrow(\mathbb{N} / n))=\alpha$. 
(6) $\iota(\mathcal{F})=\alpha+1$ implies that for every $k$ there is $m \in \mathbb{N}$ such that for every $n \geq m, \iota\left(\mathcal{F}_{\{k\}} \mid\right.$ $\mathbb{N} / n) \leq \alpha$.

3.1. Homogeneous families and admissible sets. For our study it would be very useful to have a characterization of every compact hereditary family in terms of a class of families with good structural properties allowing inductive arguments, as for example the Schreier families have. This is indeed the case for the class of homogeneous families. The following definition is modelled on the the notion of $\alpha$-uniform family of Pudlak and Rödl (see [7]).

Definition 3.2. We say that a family $\mathcal{F}$ is $\alpha$-homogeneous on $M$ ( $\alpha$ a countable ordinal) iff $\emptyset \in \mathcal{F}$ and,

(a) if $\alpha=0$, then $\mathcal{F}=\{\emptyset\}$;

(b) if $\alpha=\beta+1$, then $\mathcal{F}_{\{n\}}$ is $\beta$-homogeneous on $M / n$ for every $n \in M$;

(c) if $\alpha>0$ limit, then there is an increasing sequence $\left\{\alpha_{n}\right\}_{n \in M}$ of ordinals converging to $\alpha$ such that $\mathcal{F}_{\{n\}}$ is $\alpha_{n}$-homogeneous on $M / n$ for all $n \in M$.

$\mathcal{F}$ is called homogeneous on $M$ if it is $\alpha$-homogeneous on $M$ for some countable ordinal $\alpha$.

Recall the following well known combinatorial notion ([7]). A family $\mathcal{F}$ is $\alpha$-uniform on $M$ ( $\alpha$ a countable ordinal) iff $\mathcal{F}=\{\emptyset\}$ for $\alpha=0$ or $\mathcal{F}$ satisfies $(b)$ or $(c)$ in the other cases, where homogeneous is replaced by uniform. The relationship between uniform and homogeneous families will be exposed in the Proposition 3.6 below.

Notation. If $s, t \in \mathrm{FIN}$ we write $s \sqsubseteq t$ iff $s$ is an initial segment of $t$.

REMARK 3.3. (a) It is easy to see that the only $n$-homogeneous families on $M$ are the families of subsets of $M$ with cardinality $\leq n$, denoted by $[M]^{\leq n}$. A well known $\omega$-homogeneous family on $\mathbb{N}$ is the Schreier family, and, in general, $\omega$-homogeneous families on $M$ are of the form $\{s \subseteq M: \# s \leq f(\min s)\}$, with $f: M \rightarrow \mathbb{N}$ a unbounded and increasing mapping. Observe that all those examples are regular families.

(b) In general, an arbitrary homogeneous family is not regular. However, we will show that homogeneous families are always $\sqsubseteq$-closed, hence compact. Also, it can be shown that if $\mathcal{F}$ is a homogeneous family on $M$, there is $N \subseteq M$ such that $\mathcal{F} \mid N$ is hereditary (see [7]).

Uniform families and regular families have many properties in common. One of the most remarkable is the fact that the index of these families never decrease when taking restrictions. We expose this analogy and some others in the next proposition.

Proposition 3.4. Suppose that $\mathcal{F}$ and $\mathcal{G}$ are homogeneous (regular) families on $M$. Then:

(a) If $\iota(\mathcal{F})$ is finite, then $\mathcal{F}=[M] \leq \iota(\mathcal{F})$ if $\mathcal{F}$ is homogeneous on $M$, while $\mathcal{F} \uparrow(M / n)=$ $[M / n] \leq \iota(\mathcal{F})$ for some $n \in M$ if $\mathcal{F}$ is regular on $M$.

(b) $\mathcal{F}_{\{n\}}(n \in M)$ is homogeneous (regular) on $M / n$.

(c) If $\mathcal{F}$ is $\alpha$-homogeneous, then $\partial^{(\alpha)} \mathcal{F}=\{\emptyset\}$. Hence $\iota(\mathcal{F})=\alpha$.

(d) if $\mathcal{F}$ is $\alpha$-homogeneous (regular) on $M$ and $N \subseteq M$ then $\mathcal{F} \uparrow N$ is $\alpha$-homogeneous (regular) and $\iota(\mathcal{F} \mid N)=\iota(\mathcal{F})$ for every $N \subseteq M$.

(e) $\mathcal{F} \oplus \mathcal{G}$ and $\mathcal{F} \otimes \mathcal{G}$ are homogeneous (regular), $\iota(\mathcal{F} \oplus \mathcal{G})=\iota(\mathcal{F})+\iota(\mathcal{G})$ and $\iota(\mathcal{F} \otimes \mathcal{G})=\iota(\mathcal{F}) \iota(\mathcal{G})$.

(f) If $\iota(\mathcal{F})<\iota(\mathcal{G})$ then for every $M$ there is $N \subseteq M$ such that $\mathcal{F}|N \varsubsetneqq \mathcal{G}| N$.

Proof. Suppose first that $\mathcal{F}$ is homogeneous. (a) and (b) can be shown by an easy inductive argument.

(c): Now suppose first than $\alpha=\beta+1$. By the inductive hypothesis, for every $n \in M$, $\left(\partial^{(\beta)}(\mathcal{F})\right)_{\{n\}}=\partial^{(\beta)}\left(\mathcal{F}_{\{n\}}\right)=\{\emptyset\}$. So, $[M] \leq 1=\partial^{(\beta)}(\mathcal{F})$ (since $\partial^{(\beta)}(\mathcal{F})$ is closed and it contains all singletons $\{n\}(n \in M))$. Hence $\partial^{(\beta+1)}(\mathcal{F})=\{\emptyset\}$. Suppose now that $\alpha$ is a limit ordinal. Now by the inductive hypothesis we can conclude that for every $n \in M$,

$$
\partial^{\left(\alpha_{n}\right)}\left(\mathcal{F}_{\{n\}}\right)=\left(\partial^{\left(\alpha_{n}\right)}(\mathcal{F})\right)_{\{n\}}=\{\emptyset\},
$$

where $\alpha_{n}=\iota\left(\mathcal{F}_{\{n\}}\right)$ is such that $\left(\alpha_{n}\right)_{n}$ is increasing and with limit $\alpha$. By $(39), \emptyset \in \partial^{(\alpha)} \mathcal{F}$. If there were some $s \in \partial^{(\alpha)} \mathcal{F}, s \neq \emptyset$, then $s \in \partial^{\left(\alpha_{n}+1\right)} \mathcal{F}$ for every $n$, and hence $\partial^{\left(\alpha_{n}\right)}\left(\mathcal{F}_{\{\min s\}}\right) \neq$ $\{\emptyset\}$, a contradiction. 
(d) It follows easily by induction on $\alpha$ using $(a)$.

(e) is shown by induction on $\iota(\mathcal{G})$. (f): By Proposition 3.8, there is some $N \subseteq M$ such that either $\mathcal{F}|N \subseteq \mathcal{G}| N$ or else $\mathcal{G}|N \subseteq \mathcal{F}| N$. The second alternative is impossible since it implies that $\iota(\mathcal{F})=\iota(\mathcal{F} \mid N) \geq \iota(\mathcal{G} \mid N)=\iota(\mathcal{G})$.

Finally suppose that we are dealing with regular families. (a): First, note that there must be some $s \in \mathcal{F}$ with $|s|=\iota(\mathcal{F})$, since otherwise, $\mathcal{F} \subseteq[M]^{<\iota(\mathcal{F})}$ and so, $\iota(\mathcal{F})<\iota(\mathcal{F})$ an absurd. In a similar way one shows that $\mathcal{F} \subseteq[M]^{\leq \iota(\mathcal{F})}$. All this shows that $\mathcal{F} \uparrow(M / s)=[M / s]^{\iota(\mathcal{F})}$. (b) and (c) are clear. (d): Fix $N \subseteq M$, and let $\Theta: M \rightarrow N$ be the unique order-preserving onto mapping between these two sets. Since $\mathcal{F}$ is spreading on $M$, we obtain that $\{\Theta$ "s $: s \in \mathcal{F}\} \subseteq \mathcal{F} \mid N$; using that $\Theta " s \neq \Theta " t$ is $s \neq t$ we obtain that $\iota(\mathcal{F} \mid N) \geq \iota(\mathcal{F}) \geq i(\mathcal{F} \mid N)$, as desired. (f) follows from (d), while (e) is a consequence of Theorem 3.5 and (e) for homogeneous families.

The following result is a weaker form of Theorem II.3.22 in [7].

Theorem 3.5. [7] Suppose that $\mathcal{F}$ is a compact and hereditary family. Then there is some $M$ such that $\mathcal{F} \mid M$ is homogeneous on $M$.

Proposition 3.6. Suppose that $\mathcal{F}$ is a family of finite sets of integers. Then for every countable ordinal $\alpha$ the following conditions are equivalent:

(a) $\mathcal{F}$ is $\alpha$-homogeneous on $M$.

(b) $\mathcal{F}$ is the topological closure of an $\alpha$-uniform family on $M$.

(c) $\mathcal{F}$ is compact and the set $\mathcal{F} \sqsubseteq-\max$ of $\sqsubseteq$-maximal elements of $\mathcal{F}$ is $\alpha$-uniform on $M$. Moreover

$$
\mathcal{F}=\{s \sqsubseteq t: t \in \mathcal{F} \sqsubseteq-\max \},
$$

hence $\mathcal{F}$ is $\sqsubseteq$-hereditary, i.e. if $s \sqsubseteq t \in \mathcal{F}$ then $s \in \mathcal{F}$.

Proof. (a) implies (b): The proof is by induction on $\alpha$. If $\alpha=0$ the result is clear. Suppose that $\alpha=\beta+1$. Then for every $n \in M, \mathcal{F}_{\{n\}}$ is $\beta$-homogeneous on $M / n$. Choose $\beta$-uniform families $\mathcal{G}_{n}$ on $M / n(n \in M)$ such that for every $n \in M, \mathcal{F}_{\{n\}}=\overline{\mathcal{G}_{n}}$. Set

$$
\mathcal{G}=\left\{\{n\} \cup s: s \in \mathcal{G}_{n}\right\} .
$$

It follows readily that $\mathcal{G}_{\{n\}}=\mathcal{G}_{n}$ which yields that $\mathcal{G}$ is a $\alpha$-uniform family on $M$. To finish the proof we show that $\overline{\mathcal{G}}=\mathcal{F}$. First observe that if $s \in \mathcal{F}, n=\min s$, then ${ }_{*} s \in \mathcal{F}_{\{n\}}$. So, ${ }_{*} s \in \overline{\mathcal{G}_{n}}$, and hence

$$
s=\{n\} \cup_{*} s \in \overline{\mathcal{G}_{n} \oplus\{\{n\}\}} \subseteq \overline{\mathcal{G}} .
$$

Now suppose that $\left(s_{k}\right) \subseteq \mathcal{G}, s_{k} \rightarrow_{k} s \in \overline{\mathcal{G}}$. Going to a subsequence if necessary, we may assume that $\left(s_{k}\right)$ is a $\Delta$-sequence with root $s$, i.e. $s \sqsubseteq s_{k}$ for every $k$, and $\left(s_{k} \backslash s\right)$ is a block sequence. If $s=\emptyset$, then $s \in \mathcal{F}$ by hypothesis. Otherwise, let $n=\min s$. Then $\min s_{k}=n$ for every $k$, and hence ${ }_{*} s_{k} \in \mathcal{G}_{\{n\}}$. Hence ${ }_{*} s \in \overline{\mathcal{G}_{\{n\}}}=\mathcal{F}_{\{n\}}$, and so $s \in \mathcal{F}$. The proof if $\alpha$ is limit is similar.

(b) implies (c): Suppose that $\mathcal{F}=\overline{\mathcal{G}}$, where $\mathcal{G}$ is $\alpha$-uniform on $M$. It is not difficult to show by induction on $\alpha$ that $\mathcal{G}$ is a front on $M$ (see [7]), i.e., for every infinite $N \subseteq M$ there is some $s \in \mathcal{F}$ such that $s \sqsubseteq N$, and if $s, t \in \mathcal{F}$ and $s \sqsubseteq t$ then $s=t$. Observe that the topological closure of a front is its $\sqsubseteq$-downwards closure. Indeed, suppose that $s$ is a strict initial part of some $t \in \mathcal{F}$. For every $m>s$ consider the set $M_{m}=s \cup M / m$. Using that $\mathcal{F}$ is a front on $M$ we find $t_{m} \sqsubseteq M_{m}$ such that $t_{m} \in \mathcal{F}$, moreover $s$ has to be initial segment of every $t_{m}$. This implies that $t_{m}$ converges to $s$.

So, we have that $\mathcal{F}=\{s \sqsubseteq t: t \in \mathcal{G}\}$. It is clear that this implies that $\mathcal{F} \sqsubseteq-\max =\mathcal{G}$.

(c) implies (a): Suppose that $\mathcal{F}$ is compact and $\mathcal{F} \sqsubseteq-\max$ is $\alpha$-uniform on $M$. The proof is an easy induction on $\alpha$ using that for every $m \in M$, by 40 ), $\mathcal{F}_{\{m\}}=\left\{s \sqsubseteq t: t \in \mathcal{G}_{\{m\}}\right\}$, where $\mathcal{G}=\mathcal{F} \sqsubseteq-\max$.

The next result is the well known Ramsey property of uniform families (see [7] for a more complete explanation of the Ramsey property). 
Proposition 3.7 (Ramsey Property). Suppose that $\mathcal{B}$ is a $\alpha$-uniform family on $M$, and suppose that $\mathcal{B}=\mathcal{B}_{0} \cup \mathcal{B}_{1}$. Then there is an infinite $N \subseteq M$ such that $\mathcal{B} \mid N=\mathcal{B}_{0}\lceil N$.

Proof. Induction on $\alpha$. Given $\mathcal{B}=\mathcal{B}_{0} \cup \mathcal{B}_{1}$, using inductive hypothesis, we can find a decreasing sequence $\left(M_{k}\right)_{k}$ of infinite subsets of $M$, such that, setting $m_{k}=\min M_{k}$, for every $k, M_{k+1} \subseteq$ ${ }_{*} M_{k}$ and there is an $i_{k} \in\{0,1\}$ such that $\mathcal{B}_{\left\{m_{k}\right\}} \backslash M_{k+1}=\left(\mathcal{B}_{i_{k}}\right)_{\left\{m_{k}\right\}} \mid M_{k+1}$. Then every $N \subseteq$ $\left\{m_{k}\right\}_{k}$ for which $i_{k}$ is constant has the desired property.

As an application of this Ramsey property we obtain the following two facts.

Proposition 3.8. (a) [7], [13] Suppose that $\mathcal{F}$ and $\mathcal{G}$ are two compact and hereditary families. Then there is some infinite set $M$ such that either $\mathcal{F} \uparrow M \subseteq \mathcal{G} \mid M$ or $\mathcal{G} \mid M \subseteq \mathcal{F} \uparrow M$.

(b) Suppose that $\mathcal{F}$ is homogeneous on $M$. Then there is some $N \subseteq M$ such that $\mathcal{F} \mid N$ is hereditary.

Proof. (b): Set $\mathcal{B}=\mathcal{F} \sqsubseteq-\max$, and let $\mathcal{B}_{0}=\{s \in \mathcal{B}: \mathcal{P}(s) \nsubseteq \mathcal{F}\}, \mathcal{B}_{1}=\mathcal{B} \backslash \mathcal{B}_{0}$. By Ramsey, there is $N \subseteq M$ and $i=0,1$ such that $\mathcal{B}\left|N=\mathcal{B}_{i}\right| N$. If $i=1$ then we are done. Otherwise, fix $s \in \mathcal{B} \backslash N$ and $t \subseteq s$ such that $t \notin \mathcal{F}$. Using that $\mathcal{F} \sqsubseteq-\max$ is a front on $M$, we get $u \in \mathcal{B} \backslash N$ be such that $u \sqsubseteq t \cup(N / s)$. If $t \sqsubseteq u$ then $t \in \mathcal{F}$, which is impossible. So, $u \sqsubset t \varsubsetneqq s$. This means that for every $s \in \mathcal{B} \mid N$ there is some $t \varsubsetneqq s, t \in \mathcal{B} \mid N$. Hence $\emptyset \in \mathcal{B} \mid N$, which implies that $\mathcal{B} \mid N=\{\emptyset\}$ and so $\mathcal{F} \uparrow N=\{\emptyset\}$ is hereditary.

3.2. The basic combinatorial results. The families $\mathcal{F}$ and $\operatorname{Ad}(\mathcal{F})$ are in general different, unless $\mathcal{F}$ is spreading. Nevertheless, as it is shown in the next result, they are not so far from the topological point of view.

Proposition 3.9. Suppose that $\mathcal{F}$ is a compact hereditary family. Then for every infinite set $M$ of integers such that $\operatorname{Ad}(\mathcal{F})\lceil M$ is homogeneous on $M$,

$$
\iota(\mathcal{F}) \leq \iota(\operatorname{Ad}(\mathcal{F})\lceil M) \leq 2 \iota(\mathcal{F}) .
$$

Proof. This is done by induction on $\iota(\mathcal{F})=\lambda+n, \lambda$ limit ordinal (including $\lambda=0$ ), and $n \in \mathbb{N}$. Set $\mathcal{B}=\operatorname{Ad}(\mathcal{F}) \mid M$. Suppose first that $n=0$. Observe that in this case, by Proposition 3.1. for every $m \in M$ we have that $\partial^{(\lambda)}(\mathcal{F} \mid \mathbb{N} / m)=\{\emptyset\}$; so for every $k$, and every $m \in M$, $\lambda_{k}(m)=\iota\left(\mathcal{F}_{\{k\}}\lceil(\mathbb{N} / m))<\lambda\right.$, and $\sup \lambda_{k}(m)=\lambda$. Since for every $m \in M$ we have that $\mathcal{B}_{\{m\}}=\bigcup_{k<m} \operatorname{Ad}\left(\mathcal{F}_{\{k\}}\lceil(\mathbb{N} / m))\lceil(M / m)\right.$, by inductive hypothesis, we obtain that for every $m$, $\max _{k \leq m} \lambda_{k}(m) \leq \iota\left(\mathcal{B}_{\{m\}}\right) \leq 2 \max _{k \leq m} \lambda_{k}(m)$. This means that $\iota(\mathcal{B})=\lambda$.

Suppose now that $\iota(\mathcal{F})=\lambda+n+1$. First of all, there is some $i \in \mathbb{N}$ such that $\iota\left(\mathcal{F}_{\{i\}}\right) \geq \lambda+n$, so for every $m \in M / i, \iota\left(\mathcal{B}_{\{m\}}\right) \geq \lambda+n$, and hence, by definition of homogeneous families, $\iota(\mathcal{B}) \geq \lambda+n+1=\iota(\mathcal{F})$.

Now we work to show the other inequality $\iota(\mathcal{B}) \leq 2 \iota(\mathcal{F})=\lambda+2 n+2$. We proceed by contradiction assuming that $\iota(\mathcal{B}) \geq \lambda+2 n+3$. By Proposition 3.4 (d) we may assume that $\iota\left(\mathcal{B}_{\{m\}}\right) \geq \lambda+2 n+2$ for every $m \in M$. Let $\mathcal{G}=\mathcal{B} \sqsubseteq-\max$. Fix $m_{0} \in M$, and define the coloring $\Theta: \mathcal{G}_{\left\{m_{0}\right\}} \rightarrow\left\{0, \ldots, m_{0}\right\}$ by $\Theta(s)=k$, iff

there is some $t \in \mathcal{F}_{\{k\}}$ such that $\{k\} \cup t$ interpolates $\left\{m_{0}\right\} \cup s$.

By the Ramsey property of $\mathcal{G}_{\left\{m_{0}\right\}}$, we may assume, going to a subset if necessary, that $\Theta$ is constant with value $k_{0} \in\left\{0, \ldots, m_{0}\right\}$. Suppose first that $\iota\left(\mathcal{F}_{\left\{k_{0}\right\}}\right) \leq \lambda+n$; then by inductive hypothesis, $\iota\left(\mathcal{B}_{\left\{m_{0}\right\}}\right) \leq \lambda+2 n$, a contradiction with our assumption. So, $\iota\left(\mathcal{F}_{\left\{k_{0}\right\}}\right)=\lambda+$ $n+1$. Moreover, $\partial^{(\lambda+n+1)}\left(\mathcal{F}_{\left\{k_{0}\right\}}\right)=\emptyset$, and hence, $\partial^{(\lambda+n)} \mathcal{F}_{\left\{k_{0}\right\}} \nsubseteq\{\emptyset\}$ is finite. Let $l=$ $\max \partial^{(\lambda+n)} \mathcal{F}_{\left\{k_{0}\right\}}$. By the Ramsey property of $\mathcal{G}_{\left\{m_{0}\right\}}$ we may assume that either for every $s \in$ $\mathcal{G}_{\left\{m_{0}\right\}}$ there is some $t \in \mathcal{F}_{\left\{k_{0}\right\}}\left\lceil(\mathbb{N} / l)\right.$ who interpolates $s$, or else there is some $k_{1} \in\left\{m_{0}+1, \ldots, l\right\}$ such that for every $s \in \mathcal{G}_{\left\{m_{0}\right\}}$ there is some $t \in \mathcal{F}_{\left\{k_{0}, k_{1}\right\}}$ such that $\left\{k_{0}, k_{1}\right\} \cup t$ interpolates $\left\{m_{0}\right\} \cup$ s. In the first case, $\mathcal{B}_{\left\{m_{0}\right\}} \subseteq \operatorname{Ad}\left(\mathcal{F}_{\left\{k_{0}\right\}} \mid(\mathbb{N} / l)\right)$; since $\left.\iota\left(\mathcal{F}_{\left\{k_{0}\right\}}\right\}(\mathbb{N} / l)\right) \leq \lambda+n$, then $\iota\left(\mathcal{B}_{\left\{m_{0}\right\}}\right) \leq$ $\lambda+2 n$, a contradiction. In the second case, consider the homogeneous family $\mathcal{B}_{\left\{m_{0}, m_{1}\right\}}$, where $m_{1}=\min \left(M / k_{1}\right)$. Then $\iota\left(\mathcal{B}_{\left\{m_{0}, m_{1}\right\}}\right) \geq \lambda+2 n+1$, and $\mathcal{B}_{\left\{m_{0}, m_{1}\right\}} \subseteq \operatorname{Ad}\left(\mathcal{F}_{\left\{k_{0}, k_{1}\right\}}\left\lceil\left(\mathbb{N} / m_{1}\right)\right)\right.$. Finally notice that $\iota\left(\mathcal{F}_{\left\{k_{0}, k_{1}\right\}} \uparrow\left(\mathbb{N} / m_{1}\right)\right) \leq \lambda+n$, so $\iota\left(\mathcal{B}_{\left\{m_{0}, m_{1}\right\}}\right) \leq \lambda+2 n$, a contradiction. 
Remark 3.10. The previous result is best possible. For every limit ordinal $\lambda$ and $n \in \mathbb{N}$ there is some compact hereditary family $\mathcal{F}$ such that $\iota(\mathcal{F})=\lambda+k$ and $\iota(\operatorname{Ad}(\mathcal{F})\lceil M)=\lambda+2 k$ for every $M$, hence $\iota((A d)(\mathcal{F}) \mid M)=2 \iota(\mathcal{F})$. The families are closely related to the example 3.10 from [9]. Consider a regular family $\mathcal{B}$ on $\{2 n\}$ of index $\iota(\mathcal{B})=\lambda+k$, and let $\mathcal{F}$ be the downwards closure of $\mathcal{F}=\left\{s \cup\{k+1\}_{k \in s}: s \in \mathcal{B}\right\}$. It is not difficult to prove that $\iota(\mathcal{F})=\iota(\mathcal{B})=\lambda+k$ and that for every $M \iota((\operatorname{Ad}(\mathcal{F}))\lceil M)=\lambda+2 k$.

The next result somehow tell that we may assume that the given family $\mathcal{F}$ is indeed spreading.

Proposition 3.11. Fix an arbitrary compact hereditary family $\mathcal{F}$, and an infinite set $M$. (a) There is some regular family $\mathcal{B}$ with the same index than $\mathcal{F}$ and some $N \subseteq M$ such that every $\mathcal{B}$-admissible sequence of subsets of $N$ is also $\mathcal{F}$-admissible.

(b) For every regular family $\mathcal{B}$ on $M$ with $\iota(\mathcal{B})>\iota(\mathcal{F})$ there is some $N \subseteq M$ such that every $\mathcal{B} \sqsubseteq-\max _{\text {-admissible sequence }}\left(s_{i}\right)$ of subsets of $N$ with $\# s_{i} \geq 2$ is not $\mathcal{F}$-admissible.

Proof. Let $\mathcal{C}$ be a regular and homogeneous family on $M$ such that $\iota(\mathcal{C}) \geq \iota(\mathcal{F})=\lambda+r, \lambda$ limit (including $\lambda=0$ ) and $r \in \mathbb{N}$. Let $\mathcal{G}=\mathcal{G}(\mathcal{C})=\mathcal{C} \sqsubseteq-$ max. It follows, by Proposition 3.6, that $\mathcal{G}$ is an uniform family of $M$, as well as $[M]^{2} \otimes \mathcal{G}$. Since $\mathcal{C}$ is regular on $M$ with index $\geq \lambda+r$ we may assume that for every $s \in \mathcal{B}, \# s \geq r$. Observe that every $s \in[M]^{2} \otimes \mathcal{G}$ has a unique decomposition $s=s[0] \cup \cdots \cup s[l]$ with $s[0]<\cdots<s[l], \# s[i]=2$, and $\{\min s[i]\} \in \mathcal{G}$, so in particular $l \geq r-1$. Consider the following coloring,

$$
h_{\mathcal{C}, \mathcal{F}}:[M]^{2} \otimes \mathcal{G} \rightarrow\{0, \ldots, r, \infty\}
$$

defined for $s \in[M]^{2} \otimes \mathcal{G}$ by

$$
\begin{aligned}
h_{\mathcal{C}, \mathcal{F}}(s)=k \in\{0, \ldots r\} \quad & \text { iff } k \text { is minimal with the property that } \\
& (s[k], s[k+1], \ldots, s[r-1], s[r+1], \ldots, s[l]) \text { is } \mathcal{F} \text {-admissible, }
\end{aligned}
$$

if well defined and $h_{\mathcal{C}, \mathcal{F}}(s)=\infty$ otherwise. By Proposition 3.7 there is some $N=N(\mathcal{C}, \mathcal{F}) \subseteq M$ such that $h_{\mathcal{C}, \mathcal{F}}$ is constant on $[N]^{2} \otimes \mathcal{G} \mid N$ with value $k_{0}=k_{0}(\mathcal{C}, \mathcal{F}) \in\{0, \ldots, r, \infty\}$.

Claim. $k_{0}=0$ iff $\iota(\mathcal{F})=\iota(\mathcal{C})$.

Proof of Claim: The proof is by induction on $\iota(\mathcal{C})$. Suppose first that $k_{0}=0$, and suppose that $\iota(\mathcal{C})>\iota(\mathcal{F})$. By Proposition 3.1, we may assume, going to an infinite subset if needed, that for every $n \in N, \iota\left(\mathcal{C}_{\{n\}}\right) \geq \iota(\mathcal{F})$. Fix $n \in N$ and consider the coloring

$$
d:\left([N]^{2} \otimes\left(\mathcal{G}_{\{n\}}\right) \mid N\right) \oplus\left([N / n]^{1}\right) \rightarrow\{0, \ldots, n\}
$$

defined for $s=\{k\} \cup s[1] \cup s[2] \cup \cdots \cup s[l] \in\left([N]^{2} \otimes\left(\mathcal{G}_{\{n\}}\right)\lceil N) \oplus\left([N / n]^{1}\right)\right.$ by

$$
\begin{aligned}
d(s)=j \quad & \text { iff there is some } t \in \mathcal{F} \text { such that } \min t=j \text { and } \\
& t \text { interpolates }(\{n, \min s\}, s[1], \ldots, s[r-1], s[r+1], \ldots, s[l]) .
\end{aligned}
$$

Observe that $d$ is well defined since we are assuming that $k_{0}=0$. By the Ramsey property of the uniform family considered as domain of $d$ there is some infinite set $P \subseteq N$ such that $d$ is constant on $\left([P]^{2} \otimes\left(\mathcal{G}_{\{n\}}\right) \mid P\right) \oplus\left([P / n]^{1}\right)$ with value $j_{0} \in\{0, \ldots, n\}$. Take some $p \in P$ be such that $\left.\iota\left(\mathcal{F}_{\left\{j_{0}\right\}}\right\}(\mathbb{N} / p)\right)<\iota(\mathcal{F})$ (See Proposition 3.1 (6)). Then $k_{0}\left(\mathcal{C}_{\{n\}}\left|P, \mathcal{F}_{\left\{j_{0}\right\}}\right|(\mathbb{N} / p)\right)=0$, so, by inductive hypothesis, $\left.\iota\left(\mathcal{C}_{\{n\}}\right)=\iota\left(\mathcal{F}_{\left\{j_{0}\right\}}(\mathbb{N} / p)\right)\right)<\iota(\mathcal{F})$, a contradiction.

Now suppose that $\iota(\mathcal{C})=\iota(\mathcal{F})$. For every $1 \leq i \leq r$ set $\mathcal{C}_{i}={ }_{*} \mathcal{C}_{i-1}$ and $\mathcal{C}_{0}=\mathcal{C}$. Since $\mathcal{C}$ is regular it follows easily that $\mathcal{C}_{r}$ is regular with index $\lambda$. Consider the regular family $\mathcal{D}=[N] \leq 2 \otimes(\mathcal{C} \mid N)_{r}$ on $N$ with index $\iota(\mathcal{D})=\lambda$. By Proposition 3.9, $(\operatorname{Ad}(\mathcal{F})) \mid N \geq \lambda$, so there is some $P$ such that $\mathcal{D} \mid P \subseteq \operatorname{Ad}(\mathcal{F}) \oplus[P] \leq 1$. It readily follows that $*(\mathcal{D} \mid P) \subseteq \operatorname{Ad}(\mathcal{F})$. This shows that $k_{0} \in\{0, \ldots, r\}$. If $r=0$, then we are done. Now suppose that $r>0$. Let $q \in \mathbb{N}$ and $n_{0}, n_{1} \in N$ be such that $q<n_{0}<n_{1}$ and that $\iota\left(\mathcal{F}_{\{q\}}\left\lceil\left(\mathbb{N} / n_{1}\right)\right)=\lambda+r-1\right.$ (see Proposition 3.1). Since $\mathcal{C}$ is regular, we have that $\iota\left(\mathcal{C}_{\left\{n_{0}\right\}}\right)=\lambda+r-1=\iota\left(\mathcal{F}_{\{q\}}\left\lceil\left(\mathbb{N} / n_{1}\right)\right)\right.$. By inductive hypothesis there is some $P \subseteq N / n_{1}$ such that $h_{\mathcal{C}_{\left\{n_{0}\right\}}, \mathcal{F}_{\{q\}} \uparrow\left(\mathbb{N} / n_{1}\right)}:[P]^{2} \otimes \mathcal{G}_{\left\{n_{0}\right\}}\lceil$ $P \rightarrow\{0, \ldots, r-1, \infty\}$ is constant with value 0 . Take arbitrary $s \in[P]^{2} \otimes\left(\mathcal{G}_{\left\{n_{0}\right\}}\right)\lceil P$. Then 
$(s[0], \ldots, s[r-2], s[r], \ldots, s[l])$ is $\mathcal{F}_{\{q\}}$-admissible, so $\left(\left\{n_{0}, n_{1}\right\}, s[0], \ldots, s[r-2], s[r], \ldots, s[l]\right)$ is $\mathcal{F}$-admissible. Since $\left\{n_{0}, n_{1}\right\} \cup s \in[N]^{2} \otimes \mathcal{G} \mid N$ we obtain that $k_{0}=0$, as desired.

We work now to show (a): Fix a regular and homogeneous family $\mathcal{C}$ such that $\iota(\mathcal{C})=\iota(\mathcal{F})$ and such that $[\mathbb{N}] \leq r \subseteq \mathcal{C}$. Let $\left\{n_{i}\right\}=N=N(\mathcal{C}, \mathcal{F})$, and let

$$
\mathcal{D}=\{s \cup t \in \mathcal{C} \mid N: s<t, \# s=r \text { and } \exists k, l \in N \text { with } s<k<l<t \text { and } s \cup\{k\} \cup t \in \mathcal{C}\} .
$$

It is not difficult to see that $\mathcal{D}\left\{\left\{n_{2 i}\right\}\right.$ is a regular family on $\left\{n_{2 i}\right\}$ with the same index than $\iota(\mathcal{C})=\iota(\mathcal{F})$ (for example, by using Proposition 3.1). Now $\mathcal{B}=\operatorname{spread}\left(\mathcal{D}\left\lceil\left\{n_{2 i}\right\}\right)\right.$ and $\left\{n_{2 i}\right\}$ have property (a): Let $\left(s_{i}\right)$ be a $\mathcal{B}$-admissible sequence of finite subsets of $\left\{n_{2 i}\right\}$. By Proposition 1.7 we get that $s=\left\{\min s_{i}\right\} \in \mathcal{D}\left\lceil\left\{n_{2 i}\right\}\right.$. Write $s=t_{1} \cup t_{2}$, with $t_{1}<t_{2}$ and $\# t_{1}=r$. By definition of the family $\mathcal{D}$ there are $k<l$ in $N$ such that $t_{1}<k<l<t_{2}$ and $t_{1} \cup\{k\} \cup t_{2} \in \mathcal{C}$. Now, since $k_{0}(\mathcal{C}, \mathcal{F})=0$, it follows that $\left(s_{i}\right)$ is $\mathcal{F}$-admissible.

Finally, we proceed to prove (b): Suppose that $\mathcal{B}$ is an arbitrary regular family on $M$ with $\iota(\mathcal{B}) \geq \iota(\mathcal{F})$. Find $M^{\prime} \subseteq M$ such that $\mathcal{B} \mid M^{\prime}$ is also homogeneous on $M^{\prime}$. It is easy to see

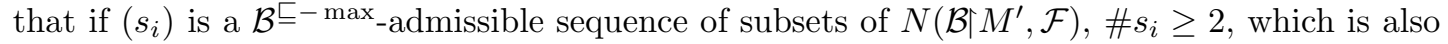
$\mathcal{F}$-admissible, then, since $\mathcal{F}$ is hereditary, $k_{0}\left(\mathcal{B} \mid M^{\prime}, \mathcal{F}\right)=0$, so by the Claim, $\iota(\mathcal{B})=\iota\left(\mathcal{B} \mid N^{\prime}\right)=$ $\iota(\mathcal{F})$. This shows $(\mathrm{b})$.

\section{BLOCK SEQUENCES OF $T\left[\left(\left(\mathcal{F}_{i}, \theta_{i}\right)_{i=1}^{r}\right)\right]$}

In this last section we show that given finitely many compact hereditary families $\left(\mathcal{F}_{i}\right)_{i=1}^{r}$ such that at least one of them has infinite index there is $1 \leq i_{0} \leq r$ such that every normalized block sequence in the space $T\left[\left(\mathcal{F}_{i}, \theta_{i}\right)_{i \in I}\right]$ has a subsequence equivalent to a subsequence of the basis of the space $T\left(\mathcal{F}_{i_{0}}, \theta_{i_{0}}\right)$. We first obtain this result for the subsequences of the basis of $T\left[\left(\mathcal{F}_{i}, \theta_{i}\right)_{i \in I}\right]$ by applying the result of the previous section, and in the sequel we extent this result for block sequences.

To obtain the result for a given block sequences $\left(x_{n}\right)_{n}$ we show first that we can pass to a subsequence $\left(x_{n}\right)_{n \in M}$ which is equivalent to the subsequence $\left(e_{p_{n}}\right)_{n \in M}, p_{n}=\min \operatorname{supp} x_{n}$, of the basis of the space $T\left([\mathbb{N}] \leq 2 \otimes \operatorname{Ad}\left(\mathcal{F}_{i_{0}}\right), \theta_{i_{0}}\right)$, for appropriate fixed $1 \leq i_{0} \leq r$. Using the results for the regular families we pass to a space $T\left(\mathcal{B}, \theta_{i_{0}}\right)$ where $\mathcal{B}$ is a regular family with $\iota(\mathcal{B})=\iota\left(\mathcal{F}_{i_{0}}\right)$ and moreover the subsequence $\left(e_{p_{n}}\right)_{n \in M}$ is equivalent in the two spaces.

Restricting the study to the families $\mathcal{S}_{\xi}$, we obtain the that if $\left(x_{n}\right)_{n},\left(y_{n}\right)_{n}$ are normalized block sequence in the space $T\left(\mathcal{S}_{\xi}, \theta\right)$ such that $x_{n}<y_{n}<x_{n+1}(n \in \mathbb{N})$ then the two sequences are equivalent.

Proposition 4.1. Fix $\left(\mathcal{F}_{i}, \theta_{i}\right)_{i=1}^{r}$ with at least one of the families with infinite index. Let $i_{0}$ be such that $\left(\mathcal{F}_{i_{0}}, \theta_{i_{0}}\right)=\max _{\leq_{\mathrm{T}}}\left\{\left(\mathcal{F}_{i}, \theta_{i}\right)\right\}_{i=1}^{r}$ (See definition 2.19). Then for every $M$ there is some $N \subseteq M$ and a regular family $\mathcal{B}$ with same index than $\mathcal{F}_{i_{0}}$ such that for every sequence $\left(a_{n}\right)_{n \in N}$ of scalars,

$$
\left\|\sum_{n \in N} a_{n} e_{n}\right\|_{\left(\mathcal{B}_{i_{0}}, \theta_{i_{0}}\right)} \leq\left\|\sum_{n \in N} a_{n} e_{n}\right\|_{\left(\mathcal{F}_{i}, \theta_{i}\right)_{i=1}^{r}} \leq 2 C\left\|\sum_{n \in N} a_{n} e_{n}\right\|_{\left(\mathcal{B}_{i_{0}}, \theta_{i_{0}}\right)} .
$$

where the constant $C$ is given in Theorem 2.1s.

Proof. By Proposition 3.11 we get $N_{0} \subseteq M$ and regular families $\mathcal{B}_{i}$, with $\iota\left(\mathcal{B}_{i}\right)=\iota\left(\mathcal{F}_{i}\right)$ $(1 \leq i \leq r)$, such that every $\mathcal{B}_{i}$-admissible sequence of subsets of $N_{0}$ is also $\mathcal{F}_{i}$-admissible. By fact 2 it follows that for every sequence $\left(a_{n}\right)_{n \in N_{0}}$ of scalars,

$$
\left\|\sum_{n \in N_{0}} a_{n} e_{n}\right\|_{\left(\mathcal{B}_{i}, \theta_{i}\right)_{i=1}^{r}} \leq\left\|\sum_{n \in N_{0}} a_{n} e_{n}\right\|_{\left(\mathcal{F}_{i}, \theta_{i}\right)_{i=1}^{r}}
$$

Counting the corresponding indexes we can find now $N_{1} \subseteq N_{0}$ such that

$$
\left[N_{1}\right] \leq 2 \otimes \operatorname{Ad}\left(\mathcal{F}_{i}\right) \mid N_{1} \subseteq\left(\mathcal{C}_{i} \mid N_{1}\right) \otimes\left[N_{1}\right]^{\leq 2} \text { for every } i \leq r,
$$


where $\mathcal{C}_{i}=\mathcal{B}_{i} \oplus\left[N_{1}\right]^{\leq 1}$ if $\iota\left(\mathcal{F}_{i}\right)<\omega, \mathcal{C}_{i}=\mathcal{B}_{i}$ otherwise. It follows from Proposition 2.1 that

$$
\left\|\sum_{n \in N_{0}} a_{n} e_{n}\right\|_{\left(\mathcal{F}_{i}, \theta_{i}\right)_{i=1}^{r}} \leq 2\left\|\sum_{n \in N} a_{n} e_{n}\right\|_{\left(\mathcal{C}_{i}, \theta_{i}\right)_{i=1}^{r}} .
$$

By Theorem 2.13, using that $\mathcal{F}_{i_{0}}$ has infinite index, there exist $N \subseteq N_{1}$ such that

$$
\left\|\sum_{n \in N} a_{n} e_{n}\right\|_{\left(\mathcal{C}_{i}, \theta_{i}\right)_{i=1}^{r}} \sim\left\|\sum_{n \in N} a_{n} e_{n}\right\|_{\left(\mathcal{B}_{i_{0}}, \theta_{i_{0}}\right)} .
$$

Since $\left\|\sum_{n \in N} a_{n} e_{n}\right\|_{\left(\mathcal{B}_{i_{0}}, \theta_{i_{0}}\right)} \leq\left\|\sum_{n \in N} a_{n} e_{n}\right\|_{\left(\mathcal{B}_{i}, \theta_{i}\right)_{i=1}^{r}}$, we get the result.

REMARK 4.2. It is worth to mention that the conclusion of the above theorem does not hold in case that all families $\mathcal{F}_{i}$ 's have finite index (see [9]).

To extent the above result to block sequences first we shall need some preparing work. The following notion is descendant of the definition of initial and partial part of a vector with respect to a tree analysis introduced in [5].

Definition 4.3. Fix compact and hereditary families $\mathcal{F}_{i}$ and real number $0<\theta_{i}<1(i \leq r)$. Let $x \in c_{00}, f \in K\left(\left(\mathcal{F}_{i}, \theta_{i}\right)_{i=1}^{r}\right)$ and $\left(f_{t}\right)_{t \in \mathcal{T}}$ a tree-analysis for $f$. Suppose that $\operatorname{supp} f \cap \operatorname{ran} x \neq$ $\emptyset$. Let $t \in \mathcal{T}$ be a $\preceq$-maximal node with respect to the property that

$$
\operatorname{supp} f_{t} \cap \operatorname{ran} x=\operatorname{supp} f \cap \operatorname{ran} x .
$$

It is clear that such $t$ exists and it is unique. Let us call it $t(x)$. Note that if $t(x)$ is not a maximal node of $\mathcal{T}$, then, by maximality of $t(x)$, there are $s_{1} \neq s_{2} \in S_{t(x)}$ such that $\operatorname{supp} f_{s_{i}} \cap \operatorname{ran} x \neq \emptyset$, for $i=1,2$. Observe that the set $S_{t}$ of immediate $\preceq$-successors of $t$ is naturally ordered according to $s<t$ iff $f_{s}<f_{t}$. Now for $t(x)$ not a maximal node, let

$$
\begin{aligned}
& s_{L}(x)=\min \left\{s \in S_{t}: \operatorname{supp} f_{s} \cap \operatorname{ran} x \neq \emptyset\right\}, \\
& s_{R}(x)=\max \left\{s \in S_{t}: \operatorname{supp} f_{s} \cap \operatorname{ran} x \neq \emptyset\right\},
\end{aligned}
$$

where both minimum and maximum are with respect to the relation $<$ on $S_{t}$.

Fix now a block sequence $\left(x_{n}\right)_{n}$. For a given $n$, let $t(n)=t\left(x_{n}\right), s_{L}(n)=s_{L}\left(x_{n}\right)$ and $s_{R}(n)=s_{R}\left(x_{n}\right)$. For $t \in \mathcal{T}$, we define recursively

$$
\begin{aligned}
& D_{t}=\bigcup_{t \preceq \mathcal{T} u}\{n: u=t(n)\}=\left\{n \in \mathbb{N}: \operatorname{supp} f_{t} \cap \operatorname{ran} x_{n}=\operatorname{supp} f \cap \operatorname{ran} x_{n}\right\}, \\
& E_{t}=D_{t} \backslash \bigcup_{s \in S_{t}} D_{s}=\{n: t=t(n)\} .
\end{aligned}
$$

For each $n$, set $q_{n}=\max \operatorname{supp} x_{n}$. Define recursively on $t \in \mathcal{T}$

$$
g_{t}=\theta_{i}\left(\sum_{n \in E_{t}} \frac{f_{t}\left(x_{n}\right)}{\theta_{i}} e_{q_{n}}^{*}+\sum_{s \in S_{t}} g_{s}\right)
$$

if $f_{t}=\theta_{i} \sum_{s \in S_{t}} f_{s}$, where $\left(f_{s}\right)_{s \in S_{t}}$ is $\mathcal{F}_{i}$-admissible.

Proposition 4.4. (a) $\operatorname{supp} g_{t}=\left\{q_{n}: n \in D_{t}\right\}$ for every $t \in \mathcal{T}$.

(b) The set $\left\{e_{q_{n}}^{*}\right\}_{n \in E_{t}} \cup\left\{g_{s}\right\}_{s \in S_{t}}$ is $[\mathbb{N}] \leq 2 \otimes \operatorname{Ad}\left(\mathcal{F}_{i}\right)$-admissible for every $t \in \mathcal{T}$ such that $f_{t}=$ $\theta_{i} \sum_{s \in S_{t}} f_{s}$.

Proof. (a) follows readily from the definitions.

(b) Suppose that $\# S_{t}>1$, otherwise there is nothing to prove. Let us observe that for every $s \in S_{t}, s$ not being the <-maximal element of $S_{t}$ and $D_{s} \neq \emptyset$,

$$
\min \operatorname{supp} f_{s} \leq \operatorname{supp} g_{s}<\min \operatorname{supp} f_{s^{+}} .
$$

The first inequality follows readily from (a).

Let us show now the last inequality. Assume otherwise that $\min \operatorname{supp} f_{s^{+}} \leq \max \operatorname{supp} g_{s}=$ $\max \left\{q_{n}: n \in D_{s}\right\}$. Then there exists $n \in D_{s}=\{m: t(m)=s\}$ such that

$$
\min \operatorname{supp} f_{s^{+}} \leq q_{n}=\max \operatorname{supp} x_{n}
$$


hence supp $f_{s^{+}} \cap \operatorname{ran} x_{n} \neq \emptyset$, a contradiction since $n \in D_{s}$. It is clear that for every $n \in E_{t}$ such that $s_{R}\left(x_{n}\right)$ it not the <-maximal element of $S_{t}$, it holds

$$
\min \operatorname{supp} f_{s_{R}\left(x_{n}\right)} \leq q_{n}<\min \operatorname{supp} f_{s_{R}\left(x_{n}\right)^{+}} \text {. }
$$

Now setting for every $s \in S_{t}, B_{s}=\operatorname{supp} g_{s} \cup\left\{q_{n}: s=s_{R}\left(x_{n}\right), n \in E_{t}\right\}$, we have that $\operatorname{supp} g_{t}=\cup_{s} B_{s}$ and from the previous observations we obtain that

$$
\min \operatorname{supp} f_{s} \leq B_{s}<\min \operatorname{supp} f_{s^{+}}
$$

Therefore from the fact that $\left\{\min \operatorname{supp} f_{s}: s \in S_{t}\right\} \in \operatorname{Ad}\left(\mathcal{F}_{i}\right)$ we get the desired result.

Proposition 4.5. (a) Fix a sequence $\left(a_{n}\right)$ of scalars. Then for every $t \in \mathcal{T}$,

$$
f_{t}\left(\sum_{n \in D_{t}} a_{n} x_{n}\right)=g_{t}\left(\sum_{n \in D_{t}} a_{n} e_{q_{n}}\right) .
$$

In particular, $f\left(\sum_{n} a_{n} x_{n}\right)=g_{\emptyset}\left(\sum_{n \in D_{\emptyset}} a_{n} e_{q_{n}}\right)$.

(b) For every $t \in \mathcal{T}, g_{t} \in \frac{1}{\theta_{0}} B\left(T\left[\left([\mathbb{N}] \leq 2 \otimes \operatorname{Ad}\left(\mathcal{F}_{i}\right), \theta_{i}\right)_{i=1}^{r}\right]^{*}\right)$, where $\theta_{0}=\min _{1 \leq i \leq r} \theta_{i}$.

(c) For every sequence $\left(a_{n}\right)$ of scalars

$$
\left\|\sum_{n} a_{n} x_{n}\right\|_{\left(\mathcal{F}_{i}, \theta_{i}\right)_{i=1}^{r}} \leq \frac{1}{\theta_{0}}\left\|\sum_{n} a_{n} e_{q_{n}}\right\|_{\left([\mathbb{N}] \leq 2 \otimes \operatorname{Ad}\left(\mathcal{F}_{i}\right), \theta_{i}\right)_{i=1}^{r}} .
$$

Proof. (a) can be shown easily by downwards induction on $t \in \mathcal{T}$. (b) follows from Proposition 4.4 (b) and the fact that the dual ball of $T\left[\left([\mathbb{N}] \leq 2 \otimes \operatorname{Ad}\left(\mathcal{F}_{i}\right), \theta_{i}\right)_{i=1}^{r}\right]$ is closed on the $([\mathbb{N}] \leq 2 \otimes$ $\left.\operatorname{Ad}\left(\mathcal{F}_{i}\right), \theta_{i}\right)$-operation (see Remark 1.4).

(c) Follows from (a) and (b).

Before we give the proof of the main result of the section we need one more auxiliary lemma.

Lemma 4.6. Fix $\left(\mathcal{F}_{i}, \theta_{i}\right)_{i=1}^{r}$ with at least one of the families with infinite index, and a normalized block sequence $\left(x_{n}\right)_{n}$ in the space $T\left[\left(\mathcal{F}_{i}, \theta_{i}\right)_{i=1}^{r}\right]$. Then for every $i_{0}$ such that $\iota\left(\mathcal{F}_{i_{0}}\right) \geq \omega$ there exists infinite set $M$ such that

$$
\left\|\sum_{n \in M} a_{n} e_{p_{n}}\right\|_{\left(\mathcal{F}_{i_{0}}, \theta_{i_{0}}\right)} \leq\left\|\sum_{n \in M} a_{n} x_{n}\right\|_{\left(\mathcal{F}_{i}, \theta_{i}\right)_{i=1}^{r}}
$$

Proof. Let $\left(x_{n}\right)$ be a normalized block sequence and set $p_{n}=\min \operatorname{supp} x_{n}$ and $P_{0}=\left\{p_{n}\right\}$. Let $M_{0}$ be an infinite set of integers and let $\mathcal{B}_{i}$ be regular families on $\mathbb{N}$ with $\iota\left(\mathcal{B}_{i}\right)=\iota\left(\mathcal{F}_{i}\right)$ such that

every $\mathcal{B}_{i}$-admissible block sequence of subsets of $\left\{p_{n}\right\}_{n \in M_{0}}$ is $\mathcal{F}_{i}$-admissible. $(1 \leq i \leq r)$

Let $M_{1}=\left\{m_{2 i}\right\}$, where $\left\{m_{i}\right\}$ is the increasing enumeration of $M_{0}$.

Claim. For every sequence of scalars $\left(a_{n}\right)_{n \in M_{1}}$,

$$
\left\|\sum_{n \in M_{1}} a_{n} e_{p_{n}}\right\|_{\left(\mathcal{B}_{i}, \theta_{i}\right)_{i=1}^{r}} \leq\left\|\sum_{n \in M_{1}} a_{n} x_{n}\right\|_{\left(\mathcal{F}_{i}, \theta_{i}\right)_{i=1}^{r}} .
$$

Proof of Claim: Choose, for every $n, \phi_{n} \in K(\mathcal{F}, \theta)$ such that $\phi_{n} x_{n} \approx 1$ and $\operatorname{supp} \phi_{n} \subseteq \operatorname{supp} x_{n}$. Define now $F: K^{M_{1}}\left(\left(\mathcal{B}_{i}, \theta_{i}\right)_{i=1}^{r}\right) \rightarrow K\left(\left(\mathcal{F}_{i}, \theta_{i}\right)_{i=1}^{r}\right)$ by $F\left(e_{p_{n}}^{*}\right)=\phi_{n}$, and extend it by $F\left(\theta_{i}\left(\psi_{0}+\right.\right.$ $\left.\left.\cdots+\psi_{n}\right)\right)=\theta_{i}\left(F\left(\psi_{0}\right)+\ldots F\left(\psi_{n}\right)\right)$ if $\left(\psi_{i}\right)_{i=0}^{n} \subseteq K^{M_{1}}\left(\left(\mathcal{B}_{i}, \theta_{i}\right)_{i=1}^{r}\right)$ is a $\mathcal{B}_{i}$-admissible block sequence $(1 \leq i \leq r) . \quad F$ is well defined: Suppose that $\left(\psi_{i}\right)_{i=0}^{n} \subseteq K^{M_{1}}\left(\left(\mathcal{B}_{i}, \theta_{i}\right)_{i=1}^{r}\right)$ is $\mathcal{B}_{i^{-}}$ admissible block sequence, and set $\min \operatorname{supp} \psi_{i}=p_{m_{2 k_{i}}}, \max \operatorname{supp} \psi_{i}=p_{m_{2 l_{i}}}(0 \leq i \leq n)$. Then we have that for every $0 \leq i \leq n$

$$
\operatorname{supp} F\left(\psi_{i}\right) \subseteq\left[p_{m_{2 k_{i}}}, p_{m_{2 l_{i}+1}}\right]
$$

Since, by (43), $\left(\left\{p_{m_{2 k_{i}}}, p_{m_{2 l_{i}+1}}\right\}\right)_{i=0}^{n}$ is $\mathcal{F}_{i}$-admissible the condition (45) yields that $\left(F\left(\psi_{i}\right)\right)_{i=0}^{n}$ is $\mathcal{F}_{i}$-admissible. It is clear now that the existence of $F$ shows the desired result. 
Let $i_{0}$ be such that $\mathcal{F}_{i_{0}}$ has infinite index. Then by Propositions 3.8 and 3.9 we can find $P \subseteq\left\{p_{n}\right\}_{n \in M_{1}}$ such that

$$
[P] \leq 2 \otimes \operatorname{Ad}\left(\mathcal{F}_{i_{0}}\right) \mid P \subseteq \mathcal{B}_{i_{0}} \otimes[P] \leq 2
$$

It follows that $\left(e_{p}\right)_{p \in P} \subseteq T\left(\mathcal{F}_{i_{0}}, \theta_{i_{0}}\right)$ and $\left(e_{p}\right)_{p \in P} \subseteq T\left(\mathcal{B}_{i_{0}}, \theta_{i_{0}}\right)$ are equivalent. This, combined with the previous claim, completes the proof.

Theorem 4.7. Fix a finite sequence $\left(\mathcal{F}_{i}, \theta_{i}\right)_{i=1}^{r}$ of compact hereditary families and real numbers such that there is some $1 \leq i \leq r$ such that $\iota\left(\mathcal{F}_{i}\right)$ is infinite. Then there is $1 \leq i_{0} \leq r$ such that every normalized block sequence $\left(x_{n}\right) \subseteq T\left[\left(\mathcal{F}_{i}, \theta_{i}\right)_{i=1}^{r}\right]$ has a subsequence $\left(x_{n}\right)_{n \in N}$ which is equivalent to the subsequence $\left(e_{p_{n}}\right)$ of the natural basis $\left(e_{n}\right)$ of $T\left(\mathcal{F}_{i_{0}}, \theta_{i_{0}}\right)$, and where $p_{n}=\min \operatorname{supp} x_{n}$.

Proof. Let $\left(x_{n}\right) \subseteq T\left[\left(\mathcal{F}_{i}, \theta_{i}\right)_{i=1}^{r}\right]$ be a normalized block sequence. By Proposition 4.5 we get

$$
\left\|\sum_{n} a_{n} x_{n}\right\|_{\left(\mathcal{F}_{i}, \theta_{i}\right)_{i=1}^{r}} \leq C\left\|\sum_{n} a_{n} e_{q_{n}}\right\|_{\left([\mathbb{N}] \leq 2 \otimes \operatorname{Ad}\left(\mathcal{F}_{i}\right), \theta_{i}\right)_{i=1}^{r}},
$$

where $C=\max _{1 \leq i \leq r} \theta_{i}^{-1}$, and $q_{n}=\max \operatorname{supp} x_{n}$ for each $n$. Find an infinite set $M$ of integers and a sequence $\left(\overline{\mathcal{G}}_{i}\right)_{i=1}^{r}$ of regular families such that for every $1 \leq i \leq r$

(a) $\operatorname{Ad}\left(\mathcal{F}_{i}\right)\lceil M$ is homogeneous on $M$,

(b) $\iota\left(\mathcal{G}_{i}\right)=\iota\left(\operatorname{Ad}\left([M] \leq 2 \otimes \operatorname{Ad}\left(\mathcal{F}_{i}\right) \mid M\right)\right)+1$, and

(c) $\operatorname{Ad}\left([M] \leq 2 \otimes \operatorname{Ad}\left(\mathcal{F}_{i}\right)\right)\left|M \subseteq \mathcal{G}_{i}\right| M$

By Proposition 2.1 we get

$$
\left\|\sum_{n \in M} a_{n} e_{q_{n}}\right\|_{\left([\mathbb{N}] \leq 2 \otimes \operatorname{Ad}\left(\mathcal{F}_{i}\right), \theta_{i}\right)_{i=1}^{r}} \leq\left\|\sum_{n \in M} a_{n} e_{q_{n}}\right\|_{\left(\mathcal{G}_{i}, \theta_{i}\right)_{i=1}^{r}} .
$$

By Theorem 2.13 there is some $N \subseteq M$ and $C \geq 1$ such that

$$
\left\|\sum_{n \in N} a_{n} e_{q_{n}}\right\|_{\left(\mathcal{G}_{i}, \theta_{i}\right)_{i=1}^{r}} \leq C\left\|\sum_{n \in N} a_{n} e_{q_{n}}\right\|_{\left(\mathcal{G}_{i_{0}}, \theta_{i_{0}}\right)},
$$

where $i_{0}$ is such that $\left(\iota\left(\mathcal{G}_{i_{0}}\right), \theta_{i_{0}}\right)=\max _{<_{\mathrm{T}}}\left\{\left(\iota\left(\mathcal{G}_{i}\right), \theta_{i}\right): 1 \leq i \leq r\right\}$. Notice that $\iota\left(\mathcal{G}_{i_{0}}\right)$ and $\iota\left(\mathcal{F}_{i_{0}}\right)$ are both infinite. By Corollary 2.3 we can find $P \subseteq N$ such that

$$
\left\|\sum_{n \in P} a_{n} e_{q_{n}}\right\|_{\left(\mathcal{G}_{i_{0}}, \theta_{i_{0}}\right)} \leq 2\left\|\sum_{n \in P} a_{n} e_{p_{n}}\right\|_{\left(\mathcal{G}_{i_{0}}, \theta_{i_{0}}\right)}
$$

where $p_{n}=\min \operatorname{supp} x_{n}$ for every $n \in P$. Since, by the choice of $M$, the $\operatorname{Ad}\left(\mathcal{F}_{i_{0}}\right) \mid M$ is homogeneous on $M$ we obtain that by Proposition 3.9 that

$$
\iota\left(\mathcal{G}_{i_{0}}\right) \leq 2 \iota\left([M]^{\leq 2} \otimes \operatorname{Ad}\left(\mathcal{F}_{i_{0}}\right) \mid M\right)+1=2 \iota\left([M]^{\leq 2}\right) \iota\left(\operatorname{Ad}\left(\mathcal{F}_{i_{0}}\right) \mid M\right)+1 \leq 8 \iota\left(\mathcal{F}_{i_{0}}\right)+1
$$

Use now Proposition 3.11 to find an infinite subset $Q \subseteq P$ and a regular family $\mathcal{B}$ with the same index than $\mathcal{F}_{i_{0}}$ such that every $\mathcal{B}$-admissible sequence of subsets of $\left\{p_{n}\right\}_{n \in Q}$ is $\mathcal{F}_{i_{0}}$-admissible.

Since $\iota\left(\mathcal{F}_{i_{0}}\right)$ is infinite and $\mathcal{G}_{i_{0}}$ and $\mathcal{B}$ are regular, the inequality (49) implies that

$$
\iota\left([\mathbb{N}] \leq 2 \otimes \mathcal{G}_{i_{0}}\right)=2 \iota\left(\mathcal{G}_{i_{0}}\right) \leq 16 \iota\left(\mathcal{F}_{i_{0}}\right)+2<\iota\left(\mathcal{F}_{i_{0}}\right) 2=\iota(\mathcal{B} \otimes[\mathbb{N}] \leq 2),
$$

so we can find an infinite $R \subseteq Q$ such that

$$
\left[\left\{p_{n}\right\}_{n \in R}\right]^{\leq 2} \otimes \mathcal{G}_{i_{0}}\left\lceil\left\{p_{n}\right\}_{n \in R} \subseteq \mathcal{B} \otimes[\mathbb{N}]^{\leq 2} .\right.
$$

Hence, by Proposition 2.1,

$$
\left\|\sum_{n \in R} a_{n} e_{p_{n}}\right\|_{\left(\mathcal{G}_{i_{0}}, \theta_{i_{0}}\right)} \leq 2\left\|\sum_{n \in R} a_{n} e_{p_{n}}\right\|_{\left(\mathcal{B}, \theta_{i_{0}}\right)},
$$

while by Lemma 4.6 we can find $S \subseteq R$ such that

$$
\left\|\sum_{n \in S} a_{n} e_{p_{n}}\right\|_{\left(\mathcal{F}_{i_{0}}, \theta_{i_{0}}\right)} \leq 2\left\|\sum_{n \in S} a_{n} x_{n}\right\|_{\left(\mathcal{F}_{i_{0}}, \theta_{i_{0}}\right)} .
$$


Putting all these inequalities together we obtain

$$
\begin{aligned}
\left\|\sum_{n \in S} a_{n} e_{p_{n}}\right\|_{\left(\mathcal{G}_{i_{0}}, \theta_{i_{0}}\right)} \leq & 2\left\|\sum_{n \in S} a_{n} e_{p_{n}}\right\|_{\left(\mathcal{B}, \theta_{i_{0}}\right)} \leq 2\left\|\sum_{n \in S} a_{n} e_{p_{n}}\right\|_{\left(\mathcal{F}_{i_{0}}, \theta_{i_{0}}\right)} \leq \\
& \leq 4\left\|\sum_{n \in S} a_{n} x_{n}\right\|_{\left(\mathcal{F}_{i_{0}}, \theta_{i_{0}}\right)} \leq 4\left\|\sum_{n \in S} a_{n} x_{n}\right\|_{\left(\mathcal{F}_{i}, \theta_{i}\right)_{i=1}^{r}} .
\end{aligned}
$$

So, $\left(x_{n}\right)_{n \in S}$ and $\left(e_{p_{n}}\right)_{n \in S} \subseteq T\left(\mathcal{F}_{i_{0}}, \theta_{i_{0}}\right)$ are equivalent, as desired.

Recall from Definition 2.9 that for a given compact and hereditary family $\mathcal{F}$ we set $\gamma(\mathcal{F})$ and $n(\mathcal{F})$ for $\iota(\mathcal{F})$ and 1 respectively if $\mathcal{F}$ has finite index, and for $\omega^{\omega^{\gamma}}$ and $n$ satisfying that $\omega^{\omega^{\gamma} n} \leq \alpha<\omega^{\omega^{\gamma}(n+1)}$, if $\mathcal{F}$ has infinite index. Using this terminology we have the following

Theorem. [9], [10] Fix $\left(\mathcal{F}_{i}, \theta_{i}\right)_{i=1}^{r}$. Let $i_{0}$ be such that $\left(\mathcal{F}_{i_{0}}, \theta_{i_{0}}\right)=\max _{\leq_{\mathrm{T}}}\left\{\left(\mathcal{F}_{i}, \theta_{i}\right)\right\}_{i=1}^{r}$, and $\mathcal{G}$ be an arbitrary regular family such that $\gamma(\mathcal{G})=\gamma\left(\mathcal{F}_{i_{0}}\right)$. Then every normalized block sequence $\left(x_{n}\right)$ of $T\left[\left(\mathcal{F}_{i}, \theta_{i}\right)_{i=1}^{r}\right]$ has a block subsequence $\left(y_{n}\right)_{n}$ equivalent to the basis of $T\left(\mathcal{G}, \theta_{i_{0}}\right)$.

Notice that the condition $\gamma(\mathcal{G})=\gamma\left(\mathcal{F}_{i_{0}}\right)$ above is equivalent to $\gamma(\mathcal{G})=\iota(\mathcal{G})=\iota\left(\mathcal{F}_{i_{0}}\right)=$ $\gamma\left(\mathcal{F}_{i_{0}}\right)$, and that $\mathcal{G}$ is equal to $[\mathbb{N}] \leq \iota(\mathcal{G})$ in a tail $\mathbb{N} / m$. Now, in the same direction of this Theorem,

Corollary 4.8. Fix $\left(\mathcal{F}_{i}, \theta_{i}\right)_{i=1}^{r}$. Let $i_{0}$ be such that $\left(\mathcal{F}_{i_{0}}, \theta_{i_{0}}\right)=\max _{\leq_{\mathrm{T}}}\left\{\left(\mathcal{F}_{i}, \theta_{i}\right)\right\}_{i=1}^{r}$. Suppose that $\mathcal{G}$ is an arbitrary compact and hereditary family. If $\gamma(\mathcal{G})=\gamma\left(\mathcal{F}_{i_{0}}\right)$, then every normalized block sequence $\left(x_{n}\right)$ of $T\left[\left(\mathcal{F}_{i}, \theta_{i}\right)_{i=1}^{r}\right]$ has a subsequence $\left(x_{n}\right)_{n \in M}$ equivalent to the subsequence $\left(e_{\min \operatorname{supp} x_{n}}\right)_{n \in M}$ of the basis of $T\left(\mathcal{G}, \theta_{i_{0}}^{n(\mathcal{B}) / n\left(\mathcal{F}_{i_{0}}\right)}\right)$.

Proof. By Theorem 4.7 it is enough to have the conclusion for subsequences of the basis of $T\left(\mathcal{F}_{i_{0}}, \theta_{i_{0}}\right)$, and by Proposition 4.1 we may assume that $\mathcal{F}_{i_{0}}$ and $\mathcal{G}$ are both regular families. Set $\iota\left(\mathcal{F}_{i_{0}}\right)=\omega^{\omega^{\alpha} m+\beta} n+\delta, \iota(\mathcal{G})=\omega^{\omega^{\bar{\alpha}} \bar{m}+\bar{\beta}} \bar{n}+\bar{\delta}$ be canonical decompositions This is possible since $\gamma\left(\mathcal{F}_{i_{0}}\right)=\gamma(\mathcal{B})$ is infinite. Moreover $\bar{\alpha}=\alpha$. Using

$$
\omega^{\omega^{\alpha} m} \leq \iota\left(\mathcal{F}_{i_{0}}\right)=\omega^{\omega^{\alpha} m+\beta} n+\delta<\omega^{\omega^{\alpha} m+\beta+1}
$$

and the corresponding inequality for $\mathcal{G}$, by Theorem 2.8 we may assume that $\iota\left(\mathcal{F}_{i_{0}}\right)=\omega^{\omega^{\alpha} m}$, and $\iota(\mathcal{G})=\omega^{\omega^{\alpha} \bar{m}}$ Now the result follows from the application of Proposition 2.6 to the families $\mathcal{F}_{i_{0}}$ and $\mathcal{G}$.

In particular for Schreier families we obtain

Corollary 4.9. Fix $\left(\mathcal{F}_{i}, \theta_{i}\right)_{i=1}^{r}$ such that at least one of the families has infinite index. Let $i_{0}$ be such that $\left(\mathcal{F}_{i_{0}}, \theta_{i_{0}}\right)=\max _{\leq_{\mathrm{T}}}\left\{\left(\mathcal{F}_{i}, \theta_{i}\right)\right\}_{i=1}^{r}$, and set $\iota\left(\mathcal{F}_{i_{0}}\right)=\omega^{\omega^{\alpha} k+\delta} m+\gamma$ in canonical form. Then every normalized block sequence $\left(x_{n}\right)$ of $T\left[\left(\mathcal{F}_{i}, \theta_{i}\right)_{i=1}^{r}\right]$ has a subsequence $\left(x_{n}\right)_{n \in M}$ equivalent to the subsequence $\left(e_{\min \operatorname{supp} x_{n}}\right)_{n \in M}$ of the basis of $T\left(\mathcal{S}_{\omega^{\alpha}}, \theta_{i_{0}}^{1 / k}\right)$.

The last result of the section concerns equivalence of block sequences in the spaces $T\left(\mathcal{S}_{\xi}, \theta\right)$.

Proposition 4.10. Let $\left(x_{n}\right),\left(y_{n}\right)$ be two normalized block sequences in the space $T\left(\mathcal{S}_{\xi}, \theta\right)$ be such that $x_{n}<y_{n}<x_{n+1}(n \in \mathbb{N})$. Then $\left(x_{n}\right)$ and $\left(y_{n}\right)$ are $24 \theta^{-2}$-equivalent.

Proof. For the proof we shall use the following two relations concerning the Schreier families $\mathcal{S}_{\xi}$, and infinite subsets $N$ of integers with $\min N \geq 3$.

$$
\begin{array}{r}
{[N]^{\leq 3} \otimes \mathcal{S}_{\xi} \subseteq \mathcal{S}_{\xi} \otimes[N]^{\leq 2}} \\
{[N]^{\leq 3} \otimes\left(\mathcal{S}_{\xi} \oplus[N]^{\leq 1}\right) \subseteq \mathcal{S}_{\xi} \otimes[N]^{\leq 3}}
\end{array}
$$

The proof of these two relations follows easily by induction on $\xi$. We show now that a normalized block sequence $\left(x_{n}\right)$ is equivalent to the subsequence $\left(e_{p_{n}}\right)_{n}$ of the basis, $p_{n}=\min \operatorname{supp} x_{n}$, and this implies the result. Without loss of generality we may assume that $p_{n} \geq 3$ for every $n$. It follows easily form the spreading property of the families $\mathcal{S}_{\xi}$ that

$$
\left\|\sum_{n} a_{n} e_{p_{n}}\right\|_{\left(\mathcal{S}_{\xi}, \theta\right)} \leq\left\|\sum_{n} a_{n} x_{n}\right\|_{\left(\mathcal{S}_{\xi}, \theta\right)}
$$


For the reverse inequality, by Proposition 4.5 we get

$$
\left\|\sum_{n} a_{n} x_{n}\right\|_{\left(\mathcal{S}_{\xi}, \theta\right)} \leq \theta^{-1}\left\|\sum_{n} a_{n} e_{q_{n}}\right\|_{\left([\mathbb{N}] \leq 2 \otimes \mathcal{S}_{\xi}, \theta\right)},
$$

where $q_{n}=\max \operatorname{supp} x_{n}$ for each $n$. By (55) and Proposition 2.1 we get

$$
\left\|\sum_{n} a_{n} e_{q_{n}}\right\|_{\left([\mathbb{N}] \leq 2 \otimes \mathcal{S}_{\xi}, \theta\right)} \leq 2\left\|\sum_{n} a_{n} e_{q_{n}}\right\|_{\left(\mathcal{S}_{\xi}, \theta\right)} .
$$

As in the proof of Corollary 2.3 we get that

$$
\left\|\sum_{n} a_{n} e_{q_{n}}\right\|_{\left(\mathcal{S}_{\xi}, \theta\right)} \leq\left\|\sum_{n} a_{n} e_{p_{n}}\right\|_{\left(\mathcal{S}_{\xi} \oplus[\mathbb{N}] \leq 1, \theta\right)} .
$$

Now by (56) and again Proposition 2.1 we get that

$$
\left\|\sum_{n} a_{n} e_{p_{n}}\right\|_{\left(\mathcal{S}_{\xi} \oplus[\mathbb{N}] \leq 1, \theta\right)} \leq 3\left\|\sum_{n} a_{n} e_{p_{n}}\right\|_{\left(\mathcal{S}_{\xi}, \theta\right)} .
$$

and this completes the proof.

4.1. Incomparability. The goal here is to turn the implication presented in Corollary 4.8 into an equivalence. So we are now going to deal with the incomparability of the Tsirelson-type spaces. The main tool to distinguish two such spaces are the special convex combinations, introduced in [5]. The following lemma provides the existence of the special convex combinations, in a more general setting than the one in [5], and it is a version of the well known Pták's Lemma (see [7] for a proof).

Lemma 4.11. Suppose that $\mathcal{F}_{0}$ and $\mathcal{F}_{1}$ are two regular families with indexes $\iota\left(\mathcal{F}_{i}\right)=\omega^{\alpha_{i}} n_{i}+\beta_{i}$, $\alpha_{i}>0, n_{i} \in \mathbb{N} \beta_{i}<\omega^{\alpha_{i}}(i=0,1)$. If $\alpha_{0}<\alpha_{1}$, then for every $\varepsilon>0$ there is a convex mean $\mu$ such that $\operatorname{supp} \mu \in \mathcal{F}_{1}$ and such that $\sup _{t \in \mathcal{F}_{0}} \sum_{n \in t} \mu(n)<\varepsilon$.

The first case where the spaces are going to be totally incomparable is if the index of one of the families is at least the $\omega$-power of the other.

Lemma 4.12. Suppose that $\mathcal{F}_{0}, \mathcal{F}_{1}$ are two regular families such that $\iota\left(\mathcal{F}_{0}\right)^{\omega} \leq \iota\left(\mathcal{F}_{1}\right)$. Then $T\left(\mathcal{F}_{0}, \theta_{0}\right)$ and $T\left(\mathcal{F}_{1}, \theta_{1}\right)$ are totally incomparable.

Proof. Suppose that the desired result does not hold. By standard arguments we may assume that there exists a normalized block sequence $\left(x_{n}\right)_{n} \in T\left(\mathcal{F}_{i}, \theta_{i}\right)$ equivalent to a normalized block sequence $\left(z_{n}\right)_{n}$ of $T\left(\mathcal{F}_{j}, \theta_{j}\right), j \neq i$. By Theorem 4.7 passing to subsequences if necessary we may assume that $\left(x_{n}\right)_{n}$ is equivalent to a subsequence $\left(e_{n}\right)_{n \in M_{i}}$ of the natural basis $\left(e_{n}\right)$ of $T\left(\mathcal{F}_{i}, \theta_{i}\right)$ and that $\left(z_{n}\right)$ is equivalent to a subsequence $\left(e_{n}\right)_{n \in M_{j}}$ of the natural basis $\left(e_{n}\right)$ of $T\left(\mathcal{F}_{j}, \theta_{j}\right)$.

For $k=0,1$, let $\varphi_{k}: M_{k} \rightarrow \mathbb{N}$ be the unique order-preserving onto mapping between $M_{k}$ and $\mathbb{N}$. Note that for $k=0,1$ the family $\phi_{k}^{-1} \mathcal{F}_{k}$ is regular on $M_{k}, \iota\left(\varphi_{k}^{-1} \mathcal{F}_{k}\right)=\iota\left(\mathcal{F}_{k}\right)$ and $\left(e_{n}\right)_{n \in M_{k}} \subseteq T\left(\mathcal{F}_{k}, \theta_{k}\right)$ is 1-equivalent to $\left(e_{n}\right)_{n \in \mathbb{N}} \subseteq T\left(\varphi_{k}^{-1} \mathcal{F}_{k}, \theta_{k}\right)$. So, without loss of generality, we may assume that $M_{1}=M_{2}=\mathbb{N}$. So, we are supposing that $\left(e_{n}\right) \subseteq T\left(\mathcal{F}_{0}, \theta_{0}\right)$ is, say, $C$-equivalent to $\left(e_{n}\right) \subseteq T\left(\mathcal{F}_{1}, \theta_{1}\right)$ i.e. for every scalars $\left(a_{n}\right)$,

$$
\frac{1}{C}\left\|\sum_{n} a_{n} e_{n}\right\|_{\left(\mathcal{F}_{0}, \theta_{0}\right)} \leq\left\|\sum_{n} a_{n} e_{n}\right\|_{\left(\mathcal{F}_{1}, \theta_{1}\right)} \leq C\left\|\sum_{n} a_{n} e_{n}\right\|_{\left(\mathcal{F}_{0}, \theta_{0}\right)}
$$

Let $l \in \mathbb{N}$ be such that $\theta_{0}^{l}<\theta_{1} /(2 C)$. By our hypothesis over the indexes, $\iota\left(\mathcal{F}_{0}\right)^{l}<\iota\left(\mathcal{F}_{1}\right)$. So, by Lemma 4.11 there is some convex mean $\mu$ such that

$$
\operatorname{supp} \mu \in \mathcal{F}_{1} \text {, and } \sum_{n \in t} \mu(n)<\frac{\theta_{1}}{2 C} \text { for every } t \in \mathcal{F}_{0}^{\otimes(l-1)} .
$$


Observe that every $\phi \in K\left(\mathcal{F}_{0}, \theta_{0}\right)$ has a decomposition $\phi=\phi_{0}+\phi_{1}$, where $\operatorname{supp} \phi_{0} \in \mathcal{F}_{0}^{\otimes(l-1)}$, $\left\|\phi_{1}\right\|_{\infty} \leq \theta^{l}$ and $\operatorname{supp} \phi_{0} \cap \operatorname{supp} \phi_{1}=\emptyset$. So, for every $\phi \in K\left(\mathcal{F}_{0}, \theta_{0}\right)$,

$$
\begin{aligned}
\left|\phi\left(\sum_{n \in s} \mu(n) e_{n}\right)\right| & =\left|\phi_{0}\left(\sum_{n \in s} \mu(n) e_{n}\right)+\phi_{1}\left(\sum_{n \in s} \mu(n) e_{n}\right)\right| \\
& \leq \sum_{n \in \operatorname{supp} \phi_{0} \cap s} \mu(n)+\left\|\phi_{1}\right\|_{\infty} \sum_{n \in s} \mu(n)<\frac{\theta_{1}}{2 C}+\theta_{0}^{l}<\frac{\theta_{1}}{C},
\end{aligned}
$$

while

$$
\left\|\sum_{n \in s} \mu(n) e_{n}\right\|_{\left(\mathcal{F}_{1}, \theta_{1}\right)} \geq \theta_{1} \sum_{n \in s} \mu(n)=\theta_{1}
$$

and so, by (57),

$$
\theta_{1} \leq\left\|\sum_{n \in s} \mu(n) e_{n}\right\|_{\left(\mathcal{F}_{1}, \theta_{1}\right)} \leq C\left\|\sum_{n \in s} \mu(n) e_{n}\right\|_{\left(\mathcal{F}_{0}, \theta_{0}\right)}<C \frac{\theta_{1}}{C}
$$

a contradiction.

The second case of totally incomparability we consider is when the two families have the same index, but the corresponding $\theta$ 's are different.

Lemma 4.13. Suppose that $\mathcal{F}_{0}$ and $\mathcal{F}_{1}$ are two regular families with same index, and suppose that $\theta_{0} \neq \theta_{1}$, and $\max \left\{\theta_{0}, \theta_{1}\right\}>1 / \iota\left(\mathcal{F}_{0}\right)$, where by convention, $1 / \alpha=0$ if $\alpha$ is an infinite ordinal. Then the corresponding spaces $T\left(\mathcal{F}_{0}, \theta_{0}\right)$ and $T\left(\mathcal{F}_{1}, \theta_{1}\right)$ are totally incomparable.

Proof. Suppose first than $\iota\left(\mathcal{F}_{0}\right)=\iota\left(\mathcal{F}_{1}\right)$ is finite. Then $T\left(\mathcal{F}_{0}, \theta_{0}\right)$ and $T\left(\mathcal{F}_{1}\right)$ are isomorphic to different classical spaces $c_{0}$ or $\ell_{p}(p>1)$, and the conclusion of the Lemma trivially holds.

Suppose that $\iota\left(\mathcal{F}_{0}\right)=\iota\left(\mathcal{F}_{1}\right)$ is infinite. As in previous lemma, we may assume that $\mathcal{F}_{0}=$ $\mathcal{F}_{1}=\mathcal{F}$ and that $\left(e_{n}\right) \subseteq T\left(\mathcal{F}, \theta_{0}\right)$ is $C$-equivalent to $\left(e_{n}\right) \subseteq T\left(\mathcal{F}, \theta_{1}\right)$, i.e. for every scalars $\left(a_{n}\right)$,

$$
\frac{1}{C}\left\|\sum_{n} a_{n} e_{n}\right\|_{\left(\mathcal{F}, \theta_{0}\right)} \leq\left\|\sum_{n} a_{n} e_{n}\right\|_{\left(\mathcal{F}, \theta_{1}\right)} \leq C\left\|\sum_{n} a_{n} e_{n}\right\|_{\left(\mathcal{F}, \theta_{0}\right)} .
$$

Suppose that $\theta_{0}<\theta_{1}$. Let $l \in \mathbb{N}, l>1$ be such that $\left(\theta_{1} / \theta_{0}\right)^{l}>2 C$. Let $\left(a_{n}\right)_{n \in s}$ be a convex mean such that $s \in \mathcal{F}^{\otimes(l)}$ and $\sum_{n \in t} a_{n}<\theta_{1}^{l} /(2 C)$ for every $t \in \mathcal{F}^{\otimes(l-1)}$. As before, any functional $\phi \in K\left(\mathcal{F}, \theta_{0}\right)$ is decomposed $\phi=\phi_{0}+\phi_{1}, \operatorname{supp} \phi_{0} \cap \operatorname{supp} \phi_{1}=\emptyset$, supp $\phi_{0} \in \mathcal{F}^{\otimes(l-1)}$ and $\left\|\phi_{1}\right\|_{\infty} \leq \theta_{0}^{l}$. Then

$$
\left|\phi\left(\sum_{n \in s} a_{n} e_{n}\right)\right|=\left|\phi_{0}\left(\sum_{n \in s} a_{n} e_{n}\right)+\phi_{1}\left(\sum_{n \in s} a_{n} e_{n}\right)\right|<\frac{\theta_{1}^{l}}{2 C}+\theta_{0}^{l}<\frac{\theta_{1}^{l}}{C}
$$

Finally, by (58),

$$
\theta_{1}^{l} \leq\left\|\sum_{n} a_{n} e_{n}\right\|_{\left(\mathcal{F}, \theta_{1}\right)} \leq C\left\|\sum_{n} a_{n} e_{n}\right\|_{\left(\mathcal{F}, \theta_{0}\right)}<C \frac{\theta_{1}^{l}}{C}
$$

a contradiction.

4.2. Main result. We collect in a single result the facts we have got so far.

Theorem 4.14 (Classification theorem). Fix two sequences $\left(\mathcal{F}_{i}, \theta_{i}\right)_{i=1}^{r}$ and $\left(\mathcal{G}_{i}, \eta_{i}\right)_{i=1}^{s}$ of pairs of compact and hereditary families and real numbers in $(0,1)$. Let $1 \leq i_{0} \leq r$ and $1 \leq j_{0} \leq$ $s$ be such that $\left(\mathcal{F}_{i_{0}}, \theta_{i_{0}}\right)=\max _{\leq_{\mathrm{T}}}\left\{\left(\mathcal{F}_{i}, \theta_{i}\right): 1 \leq i \leq r\right\}$, and $\left(\mathcal{G}_{j_{0}}, \eta_{j_{0}}\right)=\max _{\leq_{\mathrm{T}}}\left\{\left(\mathcal{G}_{i}, \eta_{i}\right)\right.$ : $1 \leq i \leq s\}$. The following are equivalent:

(a) Either

(a.1) $\gamma\left(\mathcal{F}_{i_{0}}\right), \gamma\left(\mathcal{G}_{j_{0}}\right) \geq \omega, \gamma\left(\mathcal{F}_{i_{0}}\right)=\gamma\left(\mathcal{G}_{j_{0}}\right)$ and $\theta_{i_{0}}^{n\left(\mathcal{G}_{j_{0}}\right)}=\eta_{j_{0}}^{n\left(\mathcal{F}_{i_{0}}\right)}$, or else

(a.2) both $\mathcal{F}_{i_{0}}, \mathcal{G}_{j_{0}}$ have finite index, and either

(a.2.1) $\theta_{i_{0}} \gamma\left(\mathcal{F}_{i_{0}}\right), \eta_{i_{0}} \gamma\left(\mathcal{G}_{j_{0}}\right) \leq 1$, or else

(a.2.2) $\log _{\gamma\left(\mathcal{F}_{i_{0}}\right)} \theta_{i_{0}}=\log _{\gamma\left(\mathcal{G}_{j_{0}}\right)} \eta_{j_{0}}$. 
(b) Every closed infinite dimensional subspace of $T\left[\left(\mathcal{F}_{i}, \theta_{i}\right)_{i=1}^{r}\right]$ contains a subspace isomorphic to a subspace of $T\left[\left(\mathcal{G}_{i}, \eta_{i}\right)_{i=1}^{s}\right]$.

(c) For every regular family $\mathcal{B}$ such $\gamma(\mathcal{B})=\gamma\left(\mathcal{G}_{j_{0}}\right)$ and every normalized block sequence of $T\left[\left(\mathcal{F}_{i}, \theta_{i}\right)_{i=1}^{r}\right]$ there is a block subsequence (subsequence if $\mathcal{G}_{i_{0}}$ has infinite index) equivalent to a subsequence of the natural basis of $T\left(\mathcal{B}, \eta_{j_{0}}^{n(\mathcal{B}) / n\left(\mathcal{G}_{j_{0}}\right)}\right)$.

Proof. (b) implies (c). Fix a regular family $\mathcal{B}$ with same index than $\mathcal{G}_{j_{0}}$, and fix a normalized block sequence $\left(x_{n}\right)$ of $T\left[\left(\mathcal{F}_{i}, \theta_{i}\right)_{i=1}^{r}\right]$. By (b), there is some block sequence $\left(y_{n}\right)$ of $\left(x_{n}\right)$ which is equivalent to a semi normalized block sequence $\left(z_{n}\right)$ of $T\left[\left(\mathcal{G}_{i}, \eta_{i}\right)_{i=1}^{s}\right]$. By Corollary 4.8, we can find a further block subsequence $\left(w_{n}\right)$ of $\left(z_{n}\right)$ which is equivalent to a subsequence of the natural basis of $T\left(\mathcal{B}, \eta_{j_{0}}^{1 / n\left(\mathcal{G}_{j_{0}}\right)}\right)$, as desired.

(c) implies (a). First of all, fix a regular family $\mathcal{C}$ with index $\gamma\left(\mathcal{F}_{i_{0}}\right)$. By Corollary 4.8 we know that $T\left(\left(\mathcal{F}_{i}, \theta_{i}\right)_{i=1}^{r}\right)$ is saturated by subsequences of the basis of $T\left(\mathcal{C}, \theta_{i_{0}}^{1 / n\left(\mathcal{F}_{i_{0}}\right)}\right)$. Notice that (c) implies that $T\left(\mathcal{B}, \eta_{j_{0}}^{1 / n\left(\mathcal{G}_{j_{0}}\right)}\right)$ and $T\left(\mathcal{C}, \theta_{i_{0}}^{1 / n\left(\mathcal{F}_{i_{0}}\right)}\right)$ are not totally incomparable. Suppose first than $\mathcal{G}_{j_{0}}$ has finite index. Lemma 4.12 gives that $\mathcal{F}_{i_{0}}$ has also finite index, and in particular $n\left(\mathcal{F}_{i_{0}}\right)=1$. Now (a.2) follows from the properties of $\ell_{p}$ 's and $c_{0}$.

Assume now that $\mathcal{G}_{j_{0}}$ is infinite. In this case Lemma 4.12 implies that $\gamma\left(\mathcal{F}_{i_{0}}\right)=\gamma\left(\mathcal{G}_{j_{0}}\right)$. It follows, by Corollary 4.8 that $T\left[\left(\mathcal{F}_{i}, \theta_{i}\right)_{i=1}^{r}\right]$ is saturated by subsequences of $T\left(\mathcal{B}, \theta_{i_{0}}^{1 / n\left(\mathcal{F}_{i_{0}}\right)}\right)$. Hence $T\left(\mathcal{B}, \theta_{i_{0}}^{1 / n\left(\mathcal{F}_{i_{0}}\right)}\right)$ and $T\left(\mathcal{B}, \eta_{j_{0}}^{1 / n\left(\mathcal{G}_{j_{0}}\right)}\right)$ are not totally incomparable, so by Lemma 4.13 , $\theta_{i_{0}}^{1 / n\left(\mathcal{F}_{i_{0}}\right)}=\eta_{i_{0}}^{1 / n\left(\mathcal{G}_{j_{0}}\right)}$.

(a) implies (b) follows from Corollary 4.8 .

\section{REMARK 4.15 .}

1. If the families $\mathcal{F}$ are compact but not necessarily hereditary, Theorem 4.14 is also true. The main observation is that if $\mathcal{F}$ is arbitrary compact family, there is some infinite set $M$ such that $\mathcal{F}[M]=\{s \cap M: s \in \mathcal{F}\}$ is hereditary (see [6]). This fact when applied to the family $\operatorname{Ad}(\mathcal{F})$ of $\mathcal{F}$-admissible sets guarantees to follow the arguments we use for the case of hereditary families, starting with Proposition 4.5.

2. The problem of classification of full mixed Tsirelson spaces $T\left[\left(\mathcal{F}_{i}, \theta_{i}\right)_{i=0}^{\infty}\right]$ seem rather unclear. There are several obstacles if someone wants to extend the techniques presented in this paper to the general case.

\section{REFERENCES}

[1] D. E. Alspach and S. A. Argyros, Complexity of weakly null sequences. Dissertationes Math. (Rozprawy Mat.) 321 (1992).

[2] G. Androulakis, E. Odell, Th. Schlumprecht and N. Tomczak-Jaegermann, On the structure of the spreading models of a Banach space, Canadian J. Math. 57 (2005), no. 4, 673-707.

[3] S. A. Argyros, Banach spaces of the type of Tsirelson, preprint, 1992

[4] S. A. Argyros and I. Deliyanni, Banach Spaces of The type of Tsirelson. preprint, 1992

[5] S. A. Argyros and I. Deliyanni, Examples of asymptotic $l_{1}$ Banach spaces. Trans. Amer. Math. Soc. 349 (1997), no. 3, 973-995.

[6] S. A. Argyros, G. Godefroy and H. P. Rosenthal, Descriptive set theory and Banach spaces. Handbook of the geometry of Banach spaces, Vol. 2, 1007-1069, North-Holland, Amsterdam, 2003.

[7] S. A. Argyros and S. Todorcevic, Ramsey methods in analysis. Birkhauser 2005.

[8] S. F. Bellenot, Tsirelson superspaces and $l_{p}$. J. Funct. Anal. 69 (1986), no. 2, 207-228.

[9] J. Bernués and I. Deliyanni, Families of finite subsets of $\mathbb{N}$ of low complexity and Tsirelson type spaces. Math. Nachr. 222 (2001), 15-29.

[10] J. Bernués and J. Pascual, On total incomparability of mixed Tsirelson spaces. Czechoslovak Math. J. 53 (128) (2003), no. 4, 841-859.

[11] P. Casazza and T. J. Shura, Tsirelson's space. Lecture Notes in Mathematics, 1363. Springer-Verlag, Berlin, 1989.

[12] T. Figiel and W. B. Johnson, A uniformly convex Banach space which contains no $l_{p}$. Compositio Math. 29 (1974), 179-190. 
[13] I. Gasparis, A dichotomy theorem for subsets of the power set of the natural numbers. Proc. Amer. Math. Soc. 129 (2001), no. 3, 759-764.

[14] Wacław Sierpiński, Cardinal and ordinal numbers. Second revised edition. Monografie Matematyczne, Vol. 34 Państowe Wydawnictwo Naukowe, Warsaw (1965).

[15] A. Manoussakis, A note on certain equivalent norms on Tsirelson's space. Glasg. Math. J. 46 (2004), no. $2,379-390$

[16] E. Odell and Th. Schlumprecht, A Banach space block finitely universal for monotone bases. Trans. Amer. Math. Soc. 352 (2000), no. 4, 1859-1888.

[17] E. Odell and N. Tomczak-Jaegermann, On certain equivalent norms on Tsirelson's space. Illinois J. Math. 44 (2000), no. 1, 51-71.

[18] E. Odell and N. Tomczak-Jaegermann and R. Wagner Proximity to $l_{1}$ and distortion in asymptotic $l_{1}$ spaces. J. Funct. Anal. 150 (1997), no. 1, 101-145.

[19] V. Pták, A combinatorial theorem on system of inequalities and its applications to analysis, Czech. Math. J., 84, (1959), 629-630.

[20] B. S. Tsirelson, Not every Banach space contains $\ell_{p}$ or $c_{0}$, Funct. Anal. Appl. 8 (1974), 138-141.

Equipe de Logique Mathématique, Université Paris 7- Denis Diderot, C.N.R.S. -UmR 7056, 2, Place Jussieu- Case 7012, 75251 Paris Cedex 05, France

E-mail address: abad@logique.jussieu.fr

Department of Mathematics, University of Aegean, Karlovasi, Samos, GR 83200, Greece

E-mail address: amanouss@aegean.gr 